\title{
Plant-Based Natural Fibre Reinforced Composites: A Review on Fabrication, Properties and Applications
}

\author{
Md Syduzzaman ${ }^{1}\left(\mathbb{D}\right.$, Md Abdullah Al Faruque $^{2} \mathbb{D}$, Kadir Bilisik ${ }^{1}\left(\mathbb{C}\right.$ and Maryam Naebe ${ }^{2, *}(\mathbb{C}$ \\ 1 Nano/Micro Fibre Preform Design and Composite Laboratory, Department of Textile Engineering, \\ Faculty of Engineering, Erciyes University, Kayseri 38039, Turkey; \\ sayeed33tex@gmail.com (M.S.); kadirbilisik@gmail.com (K.B.) \\ 2 Institute for Frontier Materials, Deakin University, Geelong, Victoria 3216, Australia; \\ faruqueshamim86@gmail.com \\ * Correspondence: maryam.naebe@deakin.edu.au
}

Received: 24 September 2020; Accepted: 10 October 2020; Published: 13 October 2020

\begin{abstract}
The increasing global environmental concerns and awareness of renewable green resources is continuously expanding the demand for eco-friendly, sustainable and biodegradable natural fibre reinforced composites (NFRCs). Natural fibres already occupy an important place in the composite industry due to their excellent physicochemical and mechanical properties. Natural fibres are biodegradable, biocompatible, eco-friendly and created from renewable resources. Therefore, they are extensively used in place of expensive and non-renewable synthetic fibres, such as glass fibre, carbon fibre and aramid fibre, in many applications. Additionally, the NFRCs are used in automobile, aerospace, personal protective clothing, sports and medical industries as alternatives to the petroleum-based materials. To that end, in the last few decades numerous studies have been carried out on the natural fibre reinforced composites to address the problems associated with the reinforcement fibres, polymer matrix materials and composite fabrication techniques in particular. There are still some drawbacks to the natural fibre reinforced composites (NFRCs)—for example, poor interfacial adhesion between the fibre and the polymer matrix, and poor mechanical properties of the NFRCs due to the hydrophilic nature of the natural fibres. An up-to-date holistic review facilitates a clear understanding of the behaviour of the composites along with the constituent materials. This article intends to review the research carried out on the natural fibre reinforced composites over the last few decades. Furthermore, up-to-date encyclopaedic information about the properties of the NFRCs, major challenges and potential measures to overcome those challenges along with their prospective applications have been exclusively illustrated in this review work. Natural fibres are created from plant, animal and mineral-based sources. The plant-based cellulosic natural fibres are more economical than those of the animal-based fibres. Besides, these pose no health issues, unlike mineral-based fibres. Hence, in this review, the NFRCs fabricated with the plant-based cellulosic fibres are the main focus.
\end{abstract}

Keywords: green and eco-friendly composites; natural fibre reinforced composites; cellulosic fibres; mechanical properties; applications; biodegradable and sustainable

\section{Introduction}

After energy production, which contributes greatly to carbon emissions, the textile and fashion industry is considered the second most polluting industry due to generating huge amounts of wastewater, and tonnes of textile waste that is directly buried in landfills or incinerated [1-8]. In the textile industry, production of fibres plays a major role, where fibres are classified as synthetic or man-made fibres and natural fibres. Synthetic fibres are produced from non-renewable and petroleum- 
based raw materials, which are not biodegradable $[9,10]$. On the other hand, natural fibres are fabricated from natural resources such as plants and animals, which are easily available, biodegradable, biocompatible and renewable [5,9-11]. The textile fibres are currently not only used in the traditional clothing and fashion industry but in the other industries or sectors, such as automobile, aircraft, marine, sports, and agriculture industries [1,2,12]. To satisfy these various application areas, the global production and use of synthetic fibres is significantly greater than that of natural fibres, with the view that the physicochemical properties of synthetic fibres can be modified based on the final application and the product's end-use requirement $[5,13]$. However, due to the complex chemical structures of synthetic fibres, it is difficult to reuse or recycle them after their lifetime. Therefore, they are mostly dumped into landfills or incinerated, which results in serious environmental impacts [14,15].

To overcome the environmental impacts and consequently meet the demand of textiles for different application sectors, the utilisation of natural fibre reinforced composites (NFRCs) is getting more attention in industry and in the academic research community $[9,10]$. The production and utilisation of the NFRCs will eventually enhance the eco-friendliness and sustainability of material production (Figure 1). Another reason for developing the NFRCs is their lightness, due to the lower density of natural fibres (generally ranged 1.2-1.6 g/ $\mathrm{cm}^{3}$ ) compared to the synthetic fibres (e.g., glass fibre: $2.4 \mathrm{~g} / \mathrm{cm}^{3}$ ) [16]. The NFRCs are also referred to "green composites," "ecocomposites" and "biocomposites" where natural fibres are embedded with the thermosetting or thermoplastic polymers to fabricate the composites [9-11]. The NFRCs are composed of a polymer matrix (consisting of either a petroleum-based source or a natural biopolymer) and a reinforcing material (in the form of fibres or particles) [17]. The NFRCs can be classified into three groups, green, semi-green and hybrid composites, depending on the ratio of natural materials used as the reinforcing medium [17-20]. Green composites are when both the polymer matrix and reinforcing materials are procured from natural or renewable sources (e.g., PLA and hemp) [17,21,22]. The semi-green composites are composed of natural and synthetic polymers-with more natural materials [17-20]. The hybrid composites are made of a single polymeric matrix and two or more individual synthetic or man-made fibres, such as a composite structure fabricated with glass or carbon fibres [17-20]. Although developing a 100\% bio-based composite is an ongoing attempt, it is difficult due to some undesirable properties associated with the natural fibres - e.g., high moisture absorption, the long and troublesome extraction processes and low thermal stability-which often negatively influence the ultimate mechanical properties of the composite materials $[11,23,24]$. Therefore, a feasible and tremendous effort is currently going on to develop composites wherein different percentages of natural fibres can be added to the polymeric matrices. These composites are low bio-based content composites (natural fibre content of $<20 \%$ ), medium bio-based content composites (natural fibre content of 21-50\%) and high bio-based content composites (natural fibre content of 51-90\%) [17,25,26]. 


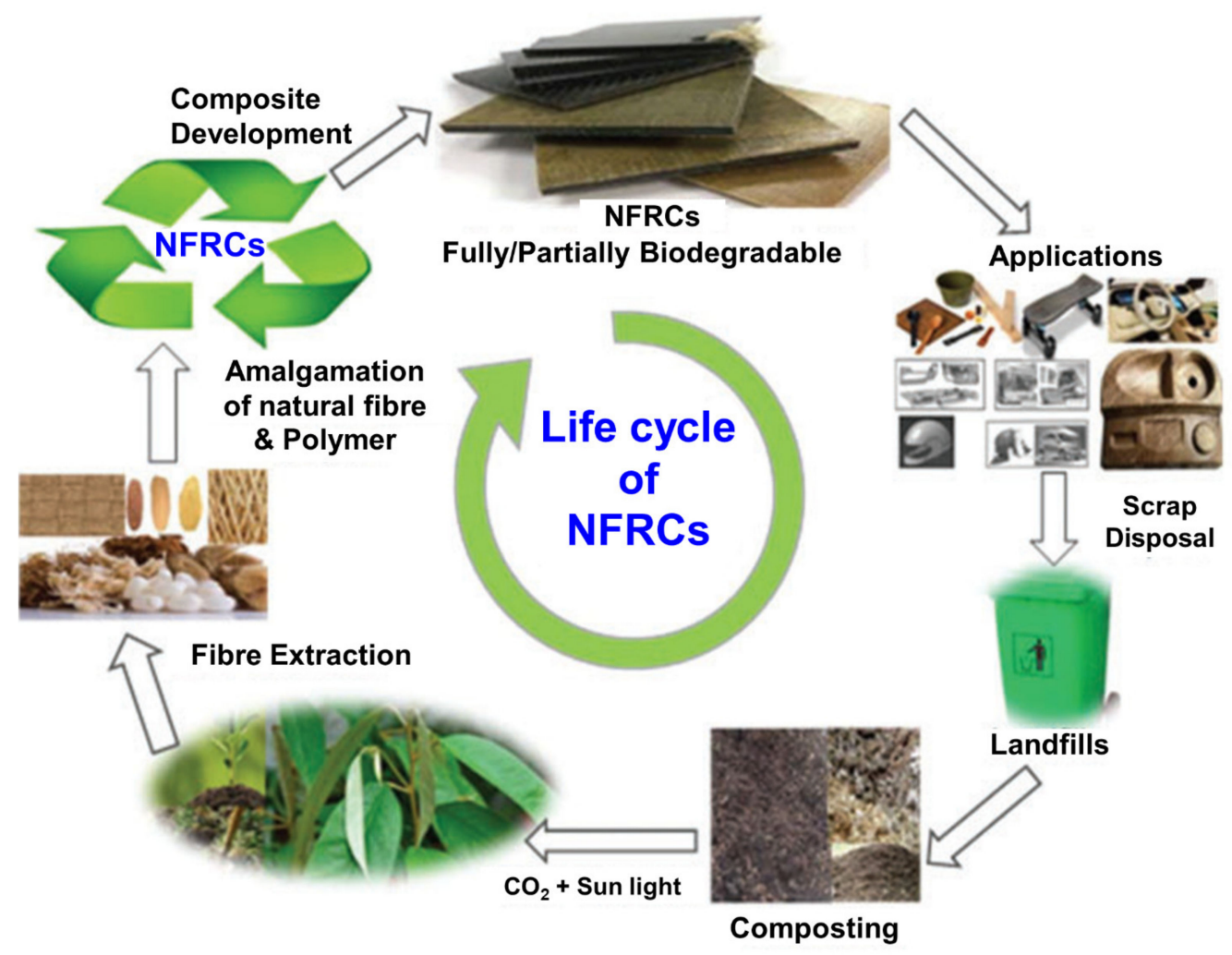

Figure 1. The life cycle of biodegradable, natural fibre reinforced composites (Reprinted with permission from [27]; Copyright 2018 SAGE).

The NFRCs are environmentally friendly, biodegradable, biocompatible, renewable and costeffective [5]. For example, these composites have the potential to replace steel in the automobile industry, which may reduce the total weight of a vehicle by around $25 \%$ and the consumption of nearly 250 million barrels of oil [5]. The NFRCs can be used in the production of bicycle frames, door and window frames, columns, ceilings and so on [28]. Besides, in recent years, along with the virgin natural fibres, the abundantly available short and non-spinnable waste natural fibres and recycled plastic materials have been used in fabricating the NFRCs. For example, waste oil plum fibres; wood flour and fibres; recyclable polymers (e.g., polyolefins); and short fibres of cotton, flax, sisal, jute, hemp and recycled plastics are used instead of virgin plastics to produce the bio-based green composites for applications wherein composites with strong mechanical properties are not required (e.g., panels, gardening products and packaging) [28-32].

Although some detailed reviews are available on the NFRCs [33-36], a single review covering the NFRCs' fabrication processes, the required properties of the natural fibres and the polymeric matrices, the detailed mechanical properties, the challenges associated with the fabrication of the NFRCs and their potential remedies with applications of the NFRCs is not present. Therefore, in this paper, we systematically review the fabrication procedures of natural fibre reinforced composites along with the physicochemical properties of the natural reinforcing materials and polymer matrices. The mechanical properties of the NFRCs, the factors affecting the mechanical properties of the NFRCs and their potential application areas are also summarised. As mentioned earlier, these composites go by many other names; we will mainly use natural fibre reinforced composites (NFRCs) or biocomposites in this review to avoid any misunderstanding of the readers. It is worth mentioning that among natural fibres, plant-based fibres are mostly used in the fabrication of NFRCs because of their price and safety. Therefore, the properties and applications of the natural fibre reinforced composites produced with the plant-based natural fibres are reviewed here. 


\section{Reinforcement and Polymer Matrix of Biocomposites}

The prime objectives of fabricating the biocomposites include but not limited to the development of a new line of fibre composites to replace the use of plastics and other synthetic fibres that will not only be eco-friendly but sustainable throughout the manufacturing process and after the useful lifetime. Generally, the term "biocomposites" refers to fabricated composite materials where two or more constituents are joined together in which at least one element is obtained from natural or bio-based resources $[17,37]$. In a broad definition, it can be said that biocomposites can be formed in between wood and non-wood based natural fibres (e.g., hard and softwood, and cotton, jute and sisal fibres), in between biopolymers and natural fibres (e.g., PLA and sisal) or even in between biopolymers and natural fibres with synthetic or man-made fibres (PLA and glass fibres) [17,37-40].

Figure 2 [41-46] shows the tree diagram of reinforcement and matrix materials associated with the fabrication of the NFRCs. NFRCs are fabricated with combinations of natural reinforcing agents and polymer matrices. The natural fibres originating from the plant, animal and mineral sources can be used directly, or in chopped fibrous strands, nonwoven mats or fabric forms.

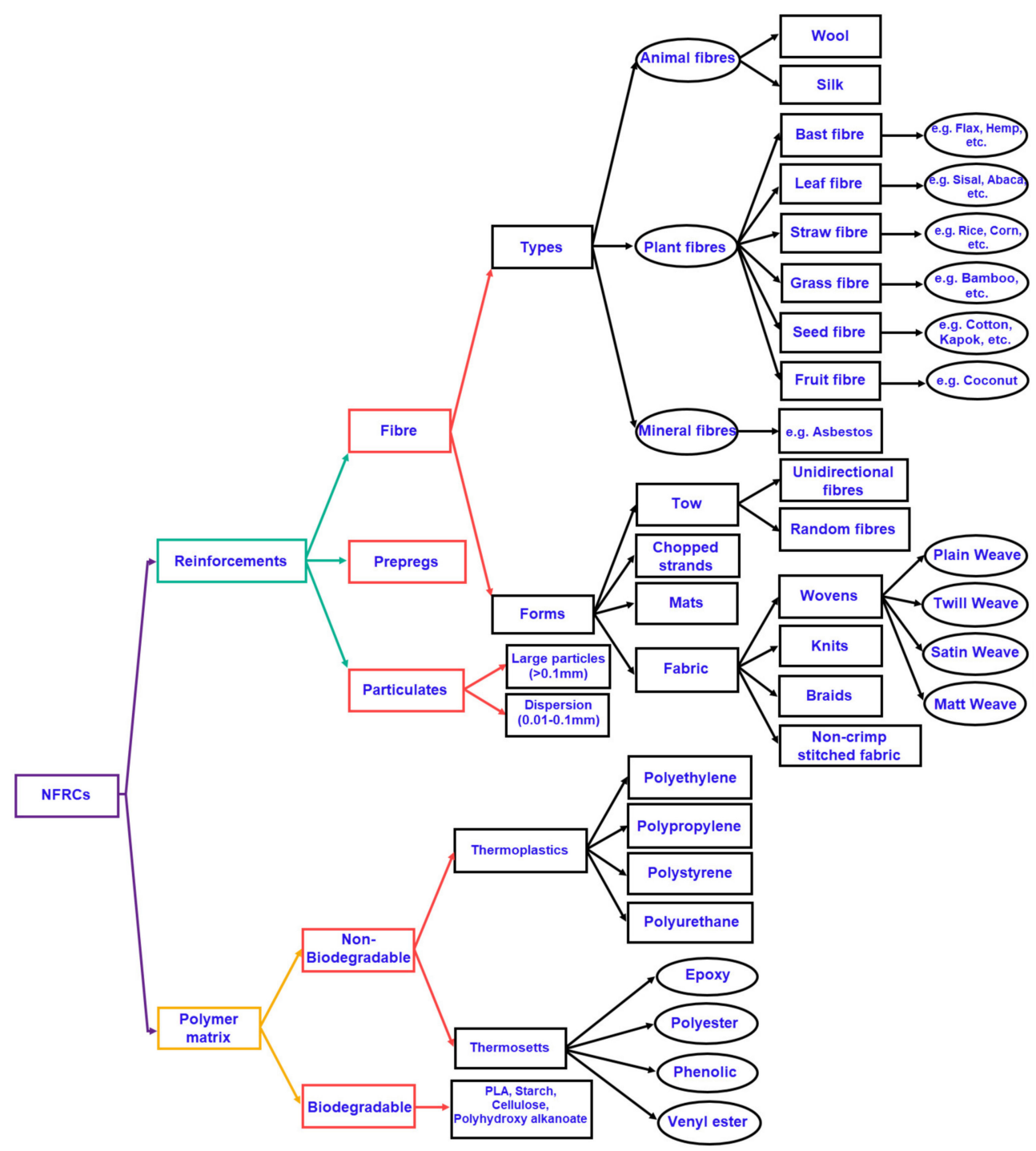

Figure 2. Composite constituent materials at a glance [41-46].

Natural fibres are broadly classified based on their origins, such as plant, animal and mineral sources (Figure 2). The plant fibres are collected from seeds (e.g., kapok, oil palm, cotton and coir), bast (e.g., ramie, hemp, flax, jute and kenaf), straw (e.g., wheat, rice and corn), wood (e.g., soft and 
hardwood), grass (e.g., bamboo and bagasse) or leaves (e.g., pineapple, sisal and abaca). The animal fibres, also known as the protein fibres and mostly used in the textile industry, are the second most widely available natural fibres after the plant-based fibres, and are obtained from sheep, alpacas, cashmere, silk, chickens and ducks [11,47-49]. Generally, the animal fibres are used in particles or chopped fibres while producing the biocomposites $[9,11,24]$. The protein fibres possess some excellent properties, such as inherent fire retardancy and thermal stability. However, the animal fibres are not widely used in the commercial production of the NFRCs due to the higher prices of the protein fibres (e.g., silk costs 2.6-40.0 US $\$ / \mathrm{kg}$ ) compared to the plant fibres (e.g., hemp costs 1.0-2.1 US\$/kg) [11,50,51]. Mineral fibres are nonmetallic and inorganic fibres produced from minerals. Asbestos and glass are the most used mineral sources for fabricating biocomposites. However, the application of asbestos fibres is currently banned due to their extensive carcinogenicity and other health issues [21,24]. As mentioned above, due to some issues associated with the animal and mineral fibres, such as cost and safety, natural plant-based fibres are mostly used in the fabrication of NFRCs, which is the focus of this study.

\subsection{Plant-Based Natural Fibres}

The most commonly used plant fibres are shown in Figure 3. The main components of the plant fibres or cellulosic fibres are cellulose, hemicellulose and lignin (Table 1) $[52,53]$. The amounts of cellulose, hemicellulose and lignin differ in the plants owing to their maturity, location of growth, environment and even species.
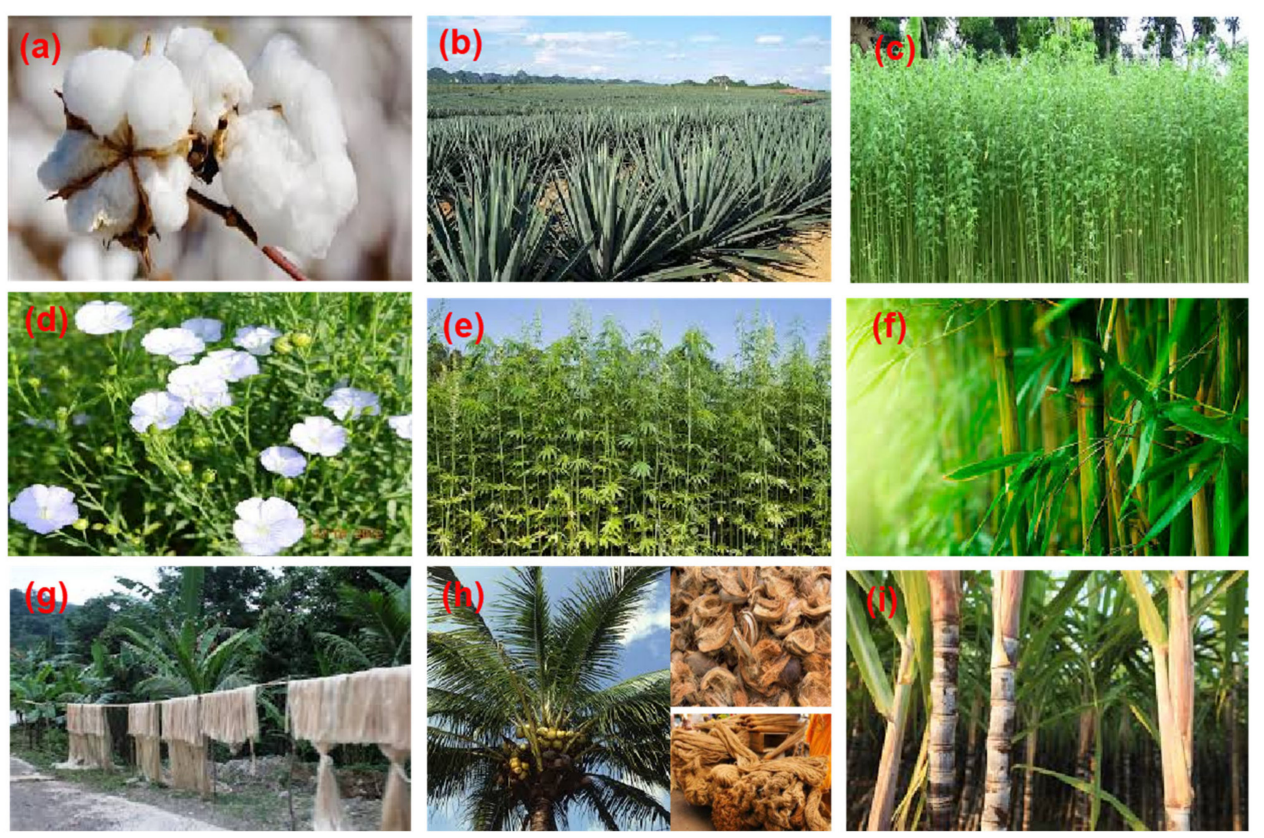

Figure 3. Natural fibre plants: (a) cotton; (b) sisal; (c) jute; (d) flax; (e) hemp; (f) bamboo; (g) banana; (h) coir; (i) sugar cane.

Cellulose, as shown in Figure 4, is a natural polysaccharide wherein D-glucopyranose rings are connected with $\beta(1 \rightarrow 4)$ glycosidic linkages $[10,53]$. All plant fibres possess a crystalline structure with a nearly $65-70 \%$ cellulose that consists of $\mathrm{C}, \mathrm{H}$ and $\mathrm{O}$ (having a common formula of $\left(\mathrm{C}_{6} \mathrm{H}_{10} \mathrm{O}_{5}\right)_{n}[10,52]$. Additionally, the final properties and characteristics of cellulosic fibres are modified with the presence of lignin and further non-cellulosic constituents. Due to the presence of a higher proportion of hydroxyl groups and hygroscopic nature, one of the important properties of the plant-based fibres is their higher moisture absorption capability, which is very often an essential requirement for the fabricated composites [22,52]. The chemical constituents and physical and mechanical properties of most widely used plant-based natural fibres are presented in Table 1. 
Table 1. Chemical constituents and physical and mechanical properties of the plant-based natural cellulosic fibres $[10,21,25,52,54-60]$.

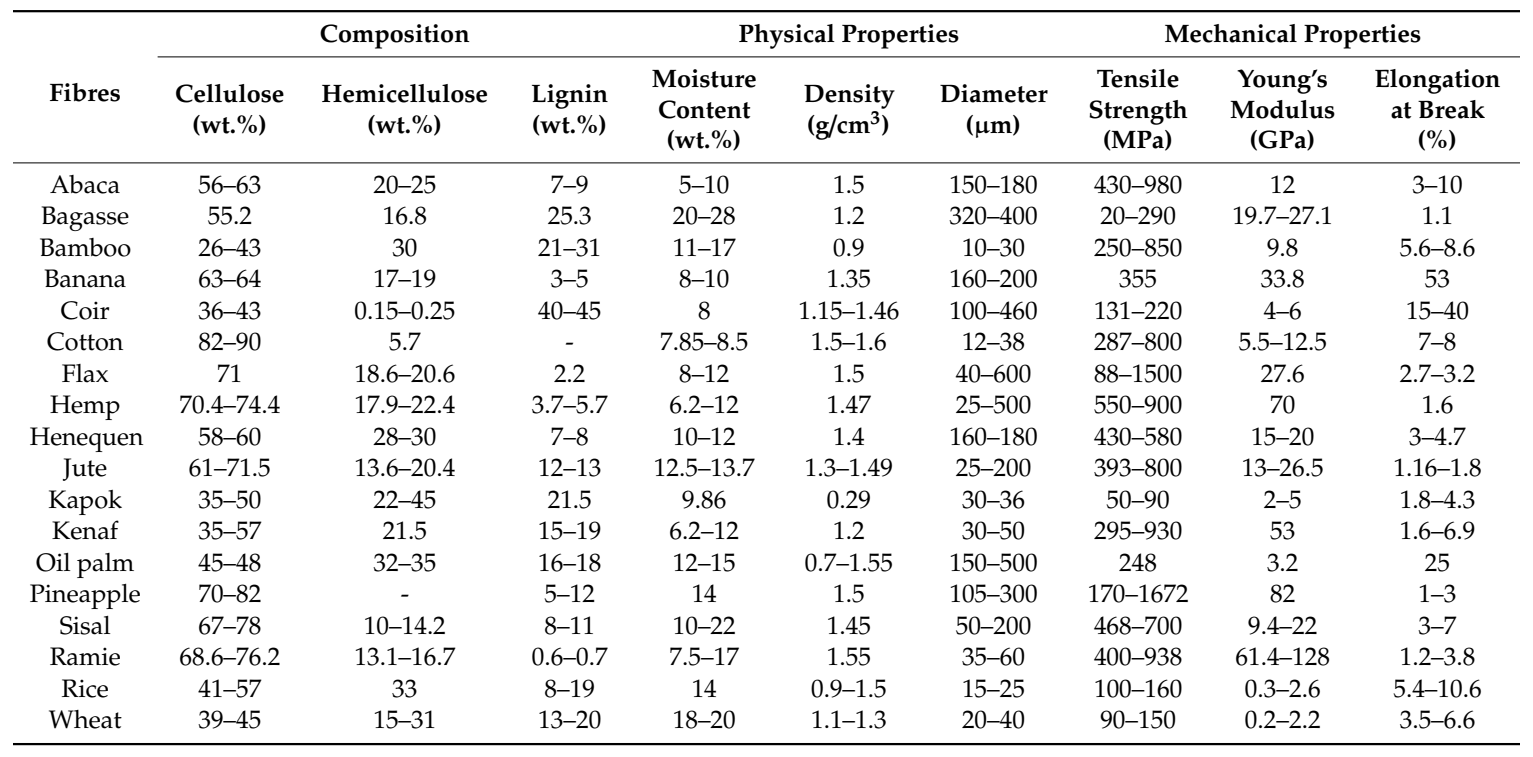

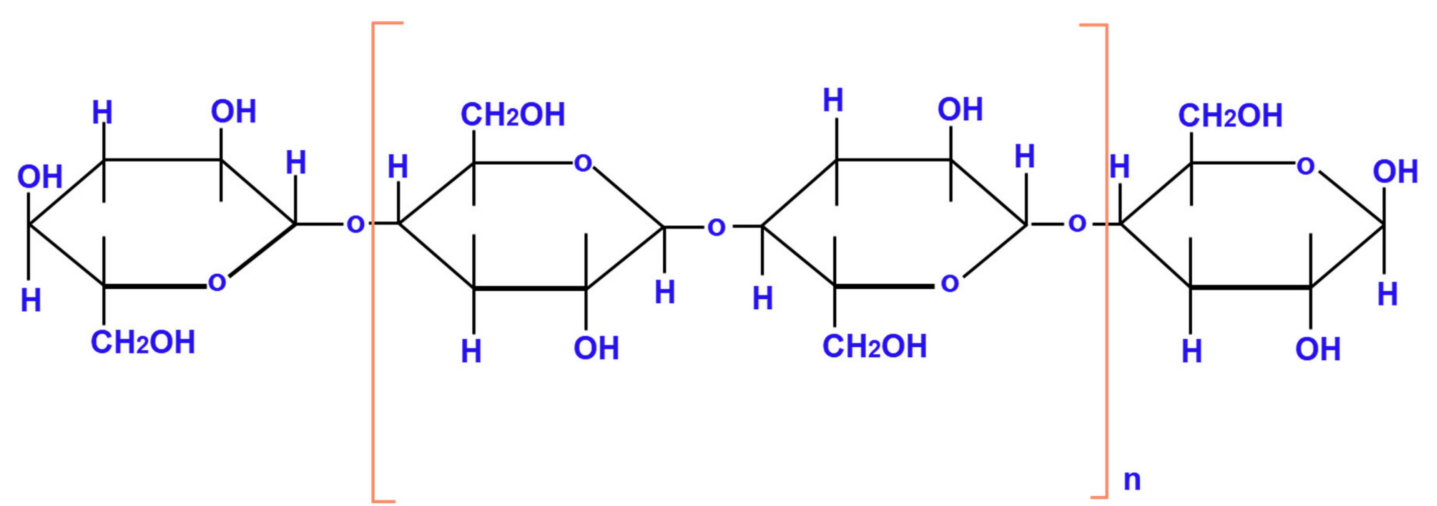

Figure 4. The chemical structure of cellulose. Reprinted with permission from [52]; Copyright 2011 Elsevier.

\subsection{Polymer Matrix}

Biocomposites are fabricated with the addition of plant-based natural fibres and polymer matrices (Figure 2). Therefore, it is very important to choose correct polymer matrices for the production of the biocomposites. Polymers are the macromolecules composed of single or many repeating units [61]. The polymeric chains in the matrix can be arranged in a unidirectional way or randomly result in a crystalline and amorphous structure, respectively. The polymers are generally divided into thermoplastic and thermosetting polymers (Figure 2) [62]. Thermoplastic polymers (e.g., polypropylene, polycarbonate, polyimides, polyethylene terephthalate) bonded with weak van der Waals forces, are widely used in manufacturing composites where both the weaker and stronger mechanical properties are required [61,62]. These polymers show outstanding toughness and resistance to wear and tear [61]. On the other hand, the thermosetting polymers (e.g., epoxy, silicone, polyurethane) are moulded into 3D networks along with strong covalent bonds and cross-linking, which makes them tough. These polymers possess higher thermal stability, creep and chemical resistance, easy processability and good wetting properties [61,63]. In general, the synthetic polymers are neither biodegradable nor flame retardant (suppressed by the addition of the nature-based polymers). Some of the key properties of the polymer matrices are illustrated in Table 2. 
Table 2. Properties of polymer matrices [61,64-66].

\begin{tabular}{|c|c|c|c|c|c|c|}
\hline Polymer & $\begin{array}{l}\text { Density } \\
\left(\mathrm{g} / \mathrm{cm}^{3}\right)\end{array}$ & $\begin{array}{l}\text { Tensile } \\
\text { Strength } \\
\text { (MPa) }\end{array}$ & $\begin{array}{l}\text { Tensile } \\
\text { Modulus } \\
\text { (GPa) }\end{array}$ & $\begin{array}{c}\text { Melting } \\
\text { Temperature } \\
\left({ }^{\circ} \mathrm{C}\right)\end{array}$ & $\begin{array}{l}\text { Thermal } \\
\text { Conductivity } \\
\left(\mathbf{W} \cdot \mathrm{m}^{-1} \cdot \mathrm{K}^{-1}\right)\end{array}$ & $\begin{array}{c}\text { Total Heat } \\
\text { Release } \\
(\mathbf{k J} / \mathrm{g})\end{array}$ \\
\hline Polyethylene (PE) & 0.93 & 15 & 0.8 & $105-115$ & $0.33-0.51$ & 41.6 \\
\hline Polypropylene (PP) & 0.92 & 40 & 1.9 & 130 & $0.1-0.2$ & 41.4 \\
\hline $\begin{array}{c}\text { Polyacrylonitrile } \\
\text { (PAN) }\end{array}$ & 1.18 & 57 & 2.7 & 300 & 1.0 & 13.3 \\
\hline Polycarbonates (PC) & 1.2 & 70 & 2.6 & 157 & 0.19 & 20.3 \\
\hline Polystyrene (PS) & 1.1 & 40 & 3 & 240 & 0.03 & 38.8 \\
\hline $\begin{array}{c}\text { Polymethyl } \\
\text { methacrylate (PMMA) }\end{array}$ & 1.18 & 47 & 2.2 & 130 & 0.20 & 24.3 \\
\hline $\begin{array}{c}\text { Polyvinyl chloride } \\
\text { (PVC) }\end{array}$ & 1.4 & 51 & 2.4 & 160 & 0.19 & 11.3 \\
\hline $\begin{array}{c}\text { Polyvinyl acetate } \\
\text { (PVA) }\end{array}$ & 1.19 & 40 & 1.7 & 200 & 0.31 & 21.6 \\
\hline Polylactic acid (PLA) & $1.2-1.4$ & 50 & 3.5 & $150-160$ & 1.13 & 14.2 \\
\hline $\begin{array}{l}\text { Polyethylene } \\
\text { terephthalate (PET) }\end{array}$ & 1.38 & 55 & 2.7 & 260 & 0.15 & 15.3 \\
\hline
\end{tabular}

\section{Fabrication of Biocomposites}

The fabrication process of the natural fibre reinforced composites (NFRCs) refers to the processes of preparing the preforms and then reinforcing the preforms with the polymer matrices [67]. The details of composite constituent (type, forms, etc.) are shown in Figure 2 [41-46]. There are a wide variety of processing techniques used to fabricate the composite materials. Application conditions for each of these techniques are quite different. Different types of composite fabrication methods can be classified according to the methods of a polymer matrix and reinforcement application to the mould, or according to the curing methods. However, all of these techniques can be broadly classified as open moulding or closed moulding techniques based on the application conditions of reinforcement and polymer matrix materials [68-73]. Open moulding is when the resin is exposed to the atmosphere during curing, and closed moulding is when the resin is not exposed to the atmosphere [74,75]. Each of these techniques has various application methods, as shown in Table 3 [42]. The composite fabrication technique is selected based on the constituent materials, the availability of the required tools and the properties required for the ultimate composite structures [76]. However, both the open and closed moulding techniques possess some benefits and drawbacks. Open moulding techniques are the most orthodox means of fabricating composites. They have been widely used because of their simplicity, low processing costs, unsophisticated techniques and variety of suitable reinforcements and laminating systems. Nevertheless, well-trained and highly skilled operators are essential to open moulding techniques to ensure the laminate quality, especially the void content and fibre volume fraction of the composite laminates [77]. On the other hand, the closed moulding techniques are preferred for making 3-dimensional composite parts of better quality, for less material waste and for perfect and aesthetic finishes of the parts $[69,78,79]$. This is mostly an automated moulding technique with reduced material, labour and waste disposal costs, and greater productivity. Compression moulding [80], extrusion moulding [81,82] and injection moulding [83-85] are the most used open moulding techniques for the thermoplastic composites. Hand layup [86,87], resin transfer moulding (RTM) [88], vacuum-assisted resin transfer moulding (VaRTM) [89] and resin film infusion (RFI) [90] are used for the production of thermosetting composites. 
Table 3. Various types of open and closed moulding techniques [42].

\begin{tabular}{cc}
\hline & Composite Moulding \\
\hline Open Moulding & Hand layup \\
- & Spray-up \\
- & Filament winding \\
Closed Moulding & Vacuum bag moulding \\
- & Resin Transfer Moulding (RTM) \\
- & Vacuum-assisted resin transfer moulding (VaRTM) \\
- & Resin film infusion, RFI \\
- & Compression moulding \\
- & Injection moulding \\
- & Pultrusion moulding \\
\hline
\end{tabular}

Figure 5a shows the two most popular open moulding techniques named hand lay-up and spray-up techniques, which are most suitable for the large composite components such as boat hulls, turbine blades, large container tanks, swimming pools and various automotive parts [91]. In the hand lay-up technique, reinforcement materials and the polymer matrix are applied manually onto an open mould surface, one by one until the desired thickness of the component is obtained by successive layers $[92,93]$. On the other hand, in the case of the spray-up technique, the reinforcement fibres in the form of chopped strands and the polymer matrices are sprayed using spray gun onto an open mould surface until the desired thickness of the composite lamination is obtained [44]. In fact, the spray-up technique is the automated version of the traditional hand lay-up technique.

Figure $5 \mathrm{~b}$ represents the VaRTM composite fabrication process. Vacuum-assisted resin transfer moulding $(\mathrm{VaRTM})$ is one of the most frequently used closed moulding processes in the fibre reinforced polymer composite industry. In this technique, the mould is filled with the reinforcement fibres and then closed, and simultaneously the air is pumped out and the polymer matrix is allowed to flow in to impregnate the fibres [94]. The reinforcement fibres are applied in a mould of the same geometry as the size and area of the required composite material [95]. The composite parts manufactured by this method possess fewer void parts with good surface finishes [96,97]. Currently, this fabrication technique is undergoing a wide variety of developments to produce more complex composite parts with the desired finishes at reasonable cost [95].

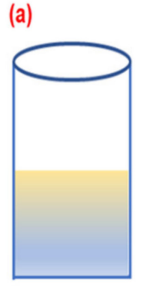

(i) Polymer matrix

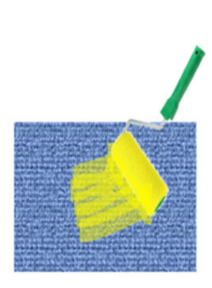

(ii) Hand layup process

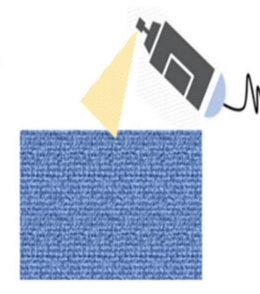

(iii) Spray-up process

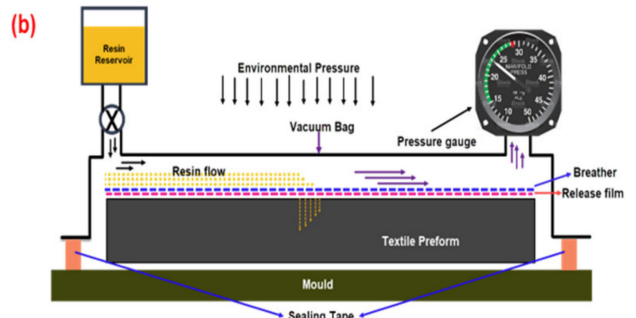

Figure 5. Composite moulding techniques. (a) Open moulding techniques: (i) polymer matrix; (ii) hand layup process; (iii) spray-up process (Reprinted with permission from [93]; Copyright 2018 Informa UK Limited). (b) Closed moulding technique: vacuum-assisted resin transfer moulding (VaRTM) (Reprinted with permission from [89]; Copyright 2012 Elsevier).

\section{Mechanical Properties of the Biocomposites}

The interfacial adhesion between the cellulosic fibre and polymer matrix sways the composite properties. The composites show better physicochemical properties if suitable interactions between the fibre and the matrix are provided [68,98]. Furthermore, fibre types, length, diameter, density, modulus, strength, fibre orientation [99-101] and weave structures [102-104] significantly control the mechanical properties of NFRCs. While NFRCs are fabricated using the hydrophilic cellulosic fibres and the hydrophobic polymer matrices, very often they result in poor interfacial adhesion [105] and 
consequently poor mechanical properties [106-108]. Therefore, investigations have been carried out to enhance the adhesion between the fibres and the polymeric matrix through adopting some techniques such as reinforcement fibre surface modification and matrix modification [109-111]. To this end, the fibres are subjected to various chemical treatments [112,113], such as alkali treatment [114-117], silane treatment [118-123], esterification [124-127], acid treatment [128] or hybridization with different fibres [129-136], before reacting with the matrix materials.

Asumani et al. [119] used alkali-treated, kenaf, short-fibre nonwoven mats to fabricate kenafpolypropylene composites by compression moulding technique and demonstrated improved mechanical properties of the composite material. The composites showed enhancements of $25 \%$, $11 \%$ and $10 \%$ for tensile strength, modulus and flexural strength, respectively. Furthermore, alkali-silane combined treatment of kenaf fibre resulted in $75 \%, 94 \%$ and $50 \%$ increases in tensile strength, modulus and flexural strength of the composite, respectively. Similarly, Wambua et al. [137] applied the randomly oriented kenaf fibres and compared the composite properties to that of glass mat-polypropylene composites. Although the tensile modulus of kenaf-polypropylene composite was slightly higher $(9.7 \%)$, the tensile strength, flexural strength, modulus and impact strength were lower $(6.7 \%, 52 \%$, $50 \%$ and $74 \%$, respectively) than those of glass fibre mat-polypropylene, which might have been due to the lower interfacial bonding strength between the fibre and matrix. Cavalcanti et al. [138] investigated the cellulose-based composite that was manufactured using the hand layup technique, where jute, curaua and sisal fibres were the reinforcing agents and epoxy was the polymeric matrix. Pure jute fibre and jute blended with curaua and sisal fibres were chemically treated with (i) alkali and (ii) combination of alkali and silane for $1 \mathrm{~h}$. Afterwards, it was found that the combined alkalisation and silanisation of jute fibre enhanced both the tensile strength $(8.4 \%)$ and flexural strength (14\%). Besides, the alkali treatment on the jute-sisal blended fibre reinforced composite demonstrated the maximal improvements. Singh et al. [139] investigated the influence of curing temperature on jute-epoxy composite properties. The jute woven preforms were used as the reinforcements and the hand layup fabrication method was applied, followed by the compression moulding technique. The authors concluded that $100{ }^{\circ} \mathrm{C}$ for flexural strength and $80^{\circ} \mathrm{C}$ for impact strength are the optimum curing temperatures that yield the maximum output. Table 4 presents the literature available on natural fibre reinforced polymer composites; it summaries the fabric/matrix modifications and their effects on associated mechanical properties. 
Table 4. Investigation of the mechanical properties of various natural fibre reinforced composites (NFRCs).

\begin{tabular}{|c|c|c|c|c|c|c|c|c|c|c|}
\hline \multirow{3}{*}{ Reinforcement Fibre } & \multirow{3}{*}{ Fibre Type } & \multirow{3}{*}{ Matrix } & \multirow{3}{*}{$\begin{array}{c}\text { Treatment (Fibre/Matrix } \\
\text { Modification) }\end{array}$} & \multirow{3}{*}{$\begin{array}{c}\text { Fabrication } \\
\text { Technique }\end{array}$} & \multicolumn{5}{|c|}{ Properties } & \multirow{3}{*}{ Ref. } \\
\hline & & & & & \multicolumn{2}{|c|}{ Tensile Properties } & \multicolumn{2}{|c|}{ Flexural Properties } & \multirow{2}{*}{$\begin{array}{c}\text { Impact } \\
\text { Strength } \\
\left(\mathrm{KJ} / \mathrm{m}^{2}\right)\end{array}$} & \\
\hline & & & & & $\begin{array}{c}\text { Strength } \\
\text { (MPa) }\end{array}$ & $\begin{array}{l}\text { Modulus } \\
\text { (GPa) }\end{array}$ & $\begin{array}{c}\text { Strength } \\
\text { (MPa) }\end{array}$ & $\begin{array}{l}\text { Modulus } \\
\text { (GPa) }\end{array}$ & & \\
\hline \multirow{2}{*}{ Kenaf fibre $(30 \%)$} & \multirow{2}{*}{$\begin{array}{l}\text { Short fibre } \\
\text { nonwoven } \\
\text { mats }\end{array}$} & \multirow{2}{*}{ Polypropylene } & Alkali treatment $(5 \% \mathrm{NaOH})$ & \multirow{2}{*}{$\begin{array}{l}\text { Compression } \\
\text { moulding }\end{array}$} & $25 \% \uparrow$ & $11 \% \uparrow$ & $10 \% \uparrow$ & - & - & \multirow{2}{*}{119} \\
\hline & & & Alkali-silane treatment $(5 \% \mathrm{NaOH})$ & & $75 \% \uparrow$ & $94 \% \uparrow$ & $50 \% \uparrow$ & - & - & \\
\hline \multirow{2}{*}{ Hemp fibre $(30 \%)$} & \multirow{2}{*}{ Aligned fibre } & \multirow{2}{*}{ Polylactic acid } & Compared to neat polylactic acid & \multirow{2}{*}{$\begin{array}{l}\text { Compression } \\
\text { moulding }\end{array}$} & $76.5 \% \uparrow$ & $201 \% \uparrow$ & $16 \% \uparrow$ & $58 \% \uparrow$ & $39.1 \% \uparrow$ & \multirow{2}{*}{140} \\
\hline & & & alkali treatment $(4 \mathrm{wt} . \% \mathrm{NaOH})$ & & $6 \% \uparrow$ & $17 \% \uparrow$ & $8 \% \uparrow$ & $14 \% \uparrow$ & $17.5 \% \uparrow$ & \\
\hline \multirow[t]{2}{*}{ Flax fibre } & $\begin{array}{l}\text { Uni-directional } \\
\text { (UD) }\end{array}$ & \multirow{2}{*}{ Epoxy } & \multirow{2}{*}{$\begin{array}{l}\text { Hybridization with UD carbon } \\
\text { fibre }\end{array}$} & \multirow{2}{*}{$\begin{array}{c}\text { Compression } \\
\text { moulding }\end{array}$} & - & - & $1.7 \% \uparrow$ & $45.1 \% \uparrow$ & & \multirow[t]{2}{*}{141} \\
\hline & Cross-ply $(\mathrm{CP})$ & & & & $282 \% \uparrow$ & $170.5 \%$ & $3.3 \% \uparrow$ & $42.9 \% \uparrow$ & & \\
\hline Sisal fibre & - & Polyethylene & Treated with $3 \%$ of stearic acid. & $\begin{array}{l}\text { Compression } \\
\text { moulding }\end{array}$ & - & - & - & - & - & {$[142]$} \\
\hline \multirow{3}{*}{ Vakka fibre } & \multirow{3}{*}{$\begin{array}{l}\text { Unidirectional } \\
\text { and } \\
\text { continuous } \\
\text { fibre }\end{array}$} & \multirow{3}{*}{ Polyester } & Compared with sisal/polyester & \multirow{3}{*}{$\begin{array}{l}\text { Hand lay-up } \\
\text { method }\end{array}$} & $32 \% \uparrow$ & $12 \% \uparrow$ & $4.4 \% \downarrow$ & $35 \% \uparrow$ & - & \multirow{3}{*}{143} \\
\hline & & & Compared with banana/polyester & & $8.4 \% \uparrow$ & $66 \% \uparrow$ & $2.6 \% \uparrow$ & $62.7 \% \uparrow$ & - & \\
\hline & & & Compared with bamboo/polyester & & $45.7 \% \downarrow$ & $20 \% \downarrow$ & $26.2 \% \downarrow$ & $9 \% \downarrow$ & - & \\
\hline \multirow{2}{*}{ Jowar fibre } & \multirow{2}{*}{$\begin{array}{l}\text { Unidirectional } \\
\text { and continuous } \\
\text { fibre }\end{array}$} & \multirow{2}{*}{ Polyester } & Compared with sisal/polyester & Hand-lay-up & $89.3 \% \uparrow$ & $44.7 \% \uparrow$ & $34.7 \% \uparrow$ & $216.1 \% \uparrow$ & - & [144] \\
\hline & & & Compared with bamboo/polyester & method & $1.8 \% \downarrow$ & $10.9 \% \uparrow$ & $4.3 \% \uparrow$ & $112.7 \% \uparrow$ & - & \\
\hline Alfa fibre & Crushed fibres & Polypropylene & Fibre esterification treatment & $\begin{array}{l}\text { Heated two roll mill } \\
\text { mixing and hot } \\
\text { press moulding } \\
\text { method }\end{array}$ & - & $35 \% \uparrow$ & - & - & - & {$[145]$} \\
\hline & & & Alkali treatment on fibre & & $8.7 \% \uparrow$ & $0.3 \% \uparrow$ & $22.3 \% \uparrow$ & $8.8 \% \uparrow$ & $1.1 \% \uparrow$ & \\
\hline Sansevieria cylindrica & Chonned fibre & Polvester & $\begin{array}{l}\text { Benzoyl peroxide treatment } \\
\text { on fibre }\end{array}$ & Compression & $13.8 \% \uparrow$ & $0.7 \% \uparrow$ & $51.9 \% \uparrow$ & $23.8 \% \uparrow$ & $5.8 \% \uparrow$ & \\
\hline fibres & & & $\begin{array}{l}\text { Potassium permanganate } \\
\text { treatment on fibre }\end{array}$ & moulding & $87.3 \% \uparrow$ & $11.9 \% \uparrow$ & $79.9 \% \uparrow$ & $37.5 \% \uparrow$ & $147.7 \% \uparrow$ & [146] \\
\hline & & & Stearic acid treatment on fibre & & $11.2 \% \uparrow$ & $0.1 \% \uparrow$ & $63.1 \% \uparrow$ & $25 \% \uparrow$ & $3.2 \% \uparrow$ & \\
\hline Coir fibre (20 wt.\%) & Crushed fibre & Polyethylene & $\begin{array}{l}\text { Sodium hydroxide }(\mathrm{NaOH}) \\
\text { treatment of fibre }\end{array}$ & $\begin{array}{l}\text { Heated two roll mill } \\
\text { mixing and hot }\end{array}$ & $6 \% \downarrow$ & $10 \% \uparrow$ & - & - & - & {$[147]$} \\
\hline & & & Silane treatment of fibre & $\begin{array}{l}\text { press moulding } \\
\text { method }\end{array}$ & $16 \% \uparrow$ & $4 \% \downarrow$ & - & - & - & \\
\hline
\end{tabular}


Table 4. Cont

\begin{tabular}{|c|c|c|c|c|c|c|c|c|c|c|}
\hline \multirow{3}{*}{ Reinforcement Fibre } & \multirow{3}{*}{ Fibre Type } & \multirow{3}{*}{ Matrix } & \multirow{3}{*}{$\begin{array}{l}\text { Treatment (Fibre/Matrix } \\
\text { Modification) }\end{array}$} & \multirow{3}{*}{$\begin{array}{c}\text { Fabrication } \\
\text { Technique }\end{array}$} & \multicolumn{5}{|c|}{ Properties } & \multirow{3}{*}{ Ref. } \\
\hline & & & & & \multicolumn{2}{|c|}{ Tensile Properties } & \multicolumn{2}{|c|}{ Flexural Properties } & \multirow{2}{*}{$\begin{array}{l}\text { Impact } \\
\text { Strength } \\
\left(\mathrm{KJ} / \mathrm{m}^{2}\right)\end{array}$} & \\
\hline & & & & & $\begin{array}{l}\text { Strength } \\
\text { (MPa) }\end{array}$ & $\begin{array}{l}\text { Modulus } \\
\text { (GPa) }\end{array}$ & $\begin{array}{l}\text { Strength } \\
\text { (MPa) }\end{array}$ & $\begin{array}{l}\text { Modulus } \\
\text { (GPa) }\end{array}$ & & \\
\hline & & & $\begin{array}{l}\text { Dodecane bromide treatment of } \\
\text { fibre }\end{array}$ & & $6 \% \uparrow$ & $24 \% \uparrow$ & - & - & - & \\
\hline Sisal fibre (30 vol.\%) & \multirow{5}{*}{$\begin{array}{l}\text { Randomly } \\
\text { oriented fibres }\end{array}$} & \multirow{5}{*}{ Polypropylene } & \multirow{5}{*}{$\begin{array}{l}\text { Compared to glass mat } \\
\text { polypropylene composites }\end{array}$} & \multirow{5}{*}{$\begin{array}{l}\text { Compression } \\
\text { moulding }\end{array}$} & $6.7 \% \uparrow$ & $14.5 \% \downarrow$ & $55 \% \downarrow$ & $59 \% \downarrow$ & $50 \% \downarrow$ & \multirow{5}{*}{ [137] } \\
\hline Kenaf fibre (30 vol.\%) & & & & & $6.7 \% \downarrow$ & $9.7 \% \uparrow$ & $52 \% \downarrow$ & $50 \% \downarrow$ & $74 \% \downarrow$ & \\
\hline Hemp fibre (30 vol.\%) & & & & & $62.5 \% \uparrow$ & $9.7 \% \uparrow$ & $10 \% \downarrow$ & $14 \% \uparrow$ & $52 \% \downarrow$ & \\
\hline Jute fibre (30 vol.\%) & & & & & $10 \% \downarrow$ & $42 \% \downarrow$ & $42 \% \downarrow$ & $36 \% \downarrow$ & $72 \% \downarrow$ & \\
\hline Coir fibre (30 vol.\%) & & & & & $68.75 \% \downarrow$ & $79 \% \downarrow$ & $53 \% \downarrow$ & $86 \% \downarrow$ & $59 \% \downarrow$ & \\
\hline Sisal & \multirow{3}{*}{$\begin{array}{l}\text { Long fibre } \\
\text { rovings }\end{array}$} & \multirow{3}{*}{ Polypropylene } & \multirow{3}{*}{$\begin{array}{l}\text { Addition of } 2 \text { wt. } \% \text { maleated } \\
\text { polypropylene }\end{array}$} & \multirow{3}{*}{$\begin{array}{l}\text { Long fibre } \\
\text { thermoplastics } \\
\text { processing and } \\
\text { compression } \\
\text { moulding }\end{array}$} & - & - & $63 \% \uparrow$ & $53.3 \% \uparrow$ & - & \multirow{3}{*}{ [75] } \\
\hline Jute & & & & & - & - & $38 \% \uparrow$ & $38.1 \% \uparrow$ & - & \\
\hline Flax & & & & & - & - & $97.5 \% \uparrow$ & $106.7 \% \uparrow$ & - & \\
\hline Sisal (20 wt.\%) & \multirow{4}{*}{$\begin{array}{l}\text { Chopped fibre } \\
\text { strands }\end{array}$} & \multirow{4}{*}{$\begin{array}{c}\text { Glycerol/ } \\
\text { thermoplastic } \\
\text { starch }\end{array}$} & \multirow{2}{*}{$\begin{array}{l}\text { Compared to neat thermoplastic } \\
\text { starch }\end{array}$} & \multirow{4}{*}{$\begin{array}{l}\text { Roll mill mixing } \\
\text { and hot press } \\
\text { moulding method }\end{array}$} & $115 \% \uparrow$ & $1410 \% \uparrow$ & - & - & - & \multirow{4}{*}{ [148] } \\
\hline Hemp (20 wt.\%) & & & & & $208 \% \uparrow$ & $1720 \% \uparrow$ & - & - & - & \\
\hline Sisal (20 wt.\%) & & & \multirow{2}{*}{$\begin{array}{l}\text { Adding latex with thermoplastic } \\
\text { starch }\end{array}$} & & $3.6 \% \downarrow$ & $1.3 \% \downarrow$ & - & - & - & \\
\hline Hemp (20 wt. $\%$ ) & & & & & $30 \% \downarrow$ & $7.7 \% \downarrow$ & - & - & - & \\
\hline \multirow[b]{2}{*}{ Kenaf fibre } & \multirow[b]{2}{*}{$\begin{array}{l}\text { Chopped fibre } \\
\text { strands }\end{array}$} & \multirow[b]{2}{*}{ Polyester } & $\begin{array}{l}\text { Magnesium hydroxide } \\
\text { impregnation with fibre }\end{array}$ & \multirow{2}{*}{$\begin{array}{c}\text { Vacuum bag resin } \\
\text { transfer moulding } \\
\text { method }\end{array}$} & $54.8 \% \uparrow$ & - & - & - & - & \multirow[b]{2}{*}{ [149] } \\
\hline & & & $\begin{array}{l}\text { Magnesium hydroxide } \\
\text { impregnation with fibre and } \\
\text { compared to the glass-fibre sheet } \\
\text { moulding compound }\end{array}$ & & $19.5 \% \downarrow$ & $21.8 \% \downarrow$ & - & - & - & \\
\hline Oil palm fibres & Fibre mats & Epoxy & Loading jute fibre & $\begin{array}{l}\text { Hand lay-up } \\
\text { technique }\end{array}$ & $68 \% \uparrow$ & $48 \% \uparrow$ & - & - & - & [150] \\
\hline Jute fibres & Cross-ply (CP) & Epoxy & Loading banana fibre & $\begin{array}{l}\text { Hand-lay-up } \\
\text { technique }\end{array}$ & $14 \% \uparrow$ & $9 \% \uparrow$ & $4.6 \% \uparrow$ & $2.4 \% \uparrow$ & $35.7 \% \uparrow$ & [151] \\
\hline
\end{tabular}


Table 4. Cont

\begin{tabular}{|c|c|c|c|c|c|c|c|c|c|c|}
\hline \multirow{3}{*}{ Reinforcement Fibre } & \multirow{3}{*}{ Fibre Type } & \multirow{3}{*}{ Matrix } & \multirow{3}{*}{$\begin{array}{c}\text { Treatment (Fibre/Matrix } \\
\text { Modification) }\end{array}$} & \multirow{3}{*}{$\begin{array}{l}\text { Fabrication } \\
\text { Technique }\end{array}$} & \multicolumn{5}{|c|}{ Properties } & \multirow{3}{*}{ Ref. } \\
\hline & & & & & \multicolumn{2}{|c|}{ Tensile Properties } & \multicolumn{2}{|c|}{ Flexural Properties } & \multirow{2}{*}{$\begin{array}{c}\text { Impact } \\
\text { Strength } \\
\left(\mathrm{KJ} / \mathrm{m}^{2}\right)\end{array}$} & \\
\hline & & & & & $\begin{array}{l}\text { Strength } \\
\text { (MPa) }\end{array}$ & $\begin{array}{l}\text { Modulus } \\
\text { (GPa) }\end{array}$ & $\begin{array}{l}\text { Strength } \\
\text { (MPa) }\end{array}$ & $\begin{array}{l}\text { Modulus } \\
\text { (GPa) }\end{array}$ & & \\
\hline \multirow{9}{*}{ Oil palm fibres } & \multirow{9}{*}{$\begin{array}{l}\text { Chopped fibre } \\
\text { strands }\end{array}$} & \multirow{9}{*}{$\begin{array}{c}\text { Phenol } \\
\text { formaldehyde }\end{array}$} & $\begin{array}{c}\text { Fibre chemical modification: } \\
\text { mercerisation }\end{array}$ & \multirow{9}{*}{$\begin{array}{l}\text { Closed and hot } \\
\text { press moulding } \\
\text { method }\end{array}$} & $5.4 \% \downarrow$ & $13 \% \uparrow$ & $53.1 \% \uparrow$ & $3.3 \% \downarrow$ & - & \multirow{9}{*}{ [152] } \\
\hline & & & $\begin{array}{l}\text { Fibre chemical modification: } \\
\text { acetylation }\end{array}$ & & $48.6 \% \downarrow$ & $30.4 \% \downarrow$ & $26.5 \% \downarrow$ & $37.7 \% \downarrow$ & - & \\
\hline & & & $\begin{array}{l}\text { Fibre chemical modification: } \\
\text { peroxide treatment }\end{array}$ & & $5.4 \% \downarrow$ & $2.2 \% \downarrow$ & $44.9 \% \uparrow$ & $29.5 \% \uparrow$ & - & \\
\hline & & & $\begin{array}{l}\text { Fibre chemical modification: } \\
\text { permanganate treatment }\end{array}$ & & $8.1 \% \uparrow$ & $4.3 \% \uparrow$ & $12.2 \% \uparrow$ & $23 \% \uparrow$ & - & \\
\hline & & & $\begin{array}{l}\text { Fibre chemical modification: } \\
\text { silanization }\end{array}$ & & $59.5 \% \downarrow$ & $39.1 \% \downarrow$ & $53.1 \% \downarrow$ & $60.7 \% \downarrow$ & - & \\
\hline & & & $\begin{array}{l}\text { Fibre chemical modification: } \\
\text { acrylation }\end{array}$ & & $51.4 \% \downarrow$ & $47.8 \% \downarrow$ & $40.8 \% \downarrow$ & $41 \% \downarrow$ & - & \\
\hline & & & $\begin{array}{l}\text { Fibre chemical modification: } \\
\text { acrylonitrile grafting }\end{array}$ & & $29.7 \% \downarrow$ & $30.4 \% \downarrow$ & $6.1 \% \uparrow$ & $18 \% \downarrow$ & - & \\
\hline & & & $\begin{array}{l}\text { Fibre chemical modification: } \\
\text { latex coating }\end{array}$ & & $64.9 \% \downarrow$ & $52.2 \% \downarrow$ & $67.3 \% \downarrow$ & $77 \% \downarrow$ & - & \\
\hline & & & Peroxide treatment on resin & & - & $8.7 \% \downarrow$ & $10.2 \% \uparrow$ & - & - & \\
\hline Coir fibre & $\begin{array}{c}\text { Randomly } \\
\text { oriented fibres }\end{array}$ & Epoxy & $\begin{array}{c}\text { Alkali treatment }(5 \mathrm{wt} . \% \mathrm{NaOH} \\
\text { solution) }\end{array}$ & $\begin{array}{l}\text { Hand lay-up } \\
\text { followed by the } \\
\text { vacuum bagging } \\
\text { technique }\end{array}$ & $17.8 \% \uparrow$ & $6.9 \% \uparrow$ & $16.8 \% \uparrow$ & $6.5 \% \uparrow$ & - & [153] \\
\hline Jute fibre & \multirow{3}{*}{$\begin{array}{l}\text { Plain woven } \\
\text { fabric }\end{array}$} & \multirow{3}{*}{ Polylactic acid } & Compared to Jute/Polypropylene & \multirow{3}{*}{ Hot-press technique } & $52.6 \% \uparrow$ & $119 \% \uparrow$ & - & $130.8 \% \uparrow$ & - & \multirow{3}{*}{ [154] } \\
\hline Sisal & & & Compared to sisal/polypropylene & & $21.6 \% \uparrow$ & $57 \% \uparrow$ & - & $84.4 \% \uparrow$ & - & \\
\hline Glass & & & Compared to glass/polypropylene & & $29.5 \% \uparrow$ & $47 \% \uparrow$ & - & $133.3 \% \uparrow$ & - & \\
\hline \multirow{2}{*}{ Jute } & \multirow{4}{*}{$\begin{array}{l}\text { Bidirectional } \\
\text { woven mat }\end{array}$} & \multirow{4}{*}{ Epoxy } & Alkalization & \multirow{4}{*}{$\begin{array}{l}\text { Hand lay-up } \\
\text { technique }\end{array}$} & - & $4.7 \% \uparrow$ & - & $9 \% \downarrow$ & $19.6 \% \uparrow$ & \multirow{4}{*}{ [138] } \\
\hline & & & Mixed (alkalization + silanization) & & $8.4 \% \uparrow$ & $9.6 \% \uparrow$ & - & $14 \% \downarrow$ & & \\
\hline \multirow{2}{*}{ Jute + Curaua } & & & Alkalization & & $9.2 \% \downarrow$ & $18.8 \% \downarrow$ & $13 \% \downarrow$ & $12 \% \downarrow$ & $25.4 \% \downarrow$ & \\
\hline & & & Mixed (alkalization + silanization) & & $1.3 \% \downarrow$ & - & $22 \% \uparrow$ & $75 \% \uparrow$ & - & \\
\hline
\end{tabular}


Table 4. Cont

\begin{tabular}{|c|c|c|c|c|c|c|c|c|c|c|}
\hline \multirow{3}{*}{ Reinforcement Fibre } & \multirow{3}{*}{ Fibre Type } & \multirow{3}{*}{ Matrix } & \multirow{3}{*}{$\begin{array}{c}\text { Treatment (Fibre/Matrix } \\
\text { Modification) }\end{array}$} & \multirow{3}{*}{$\begin{array}{l}\text { Fabrication } \\
\text { Technique }\end{array}$} & \multicolumn{5}{|c|}{ Properties } & \multirow{3}{*}{ Ref. } \\
\hline & & & & & \multicolumn{2}{|c|}{ Tensile Properties } & \multicolumn{2}{|c|}{ Flexural Properties } & \multirow{2}{*}{$\begin{array}{l}\text { Impact } \\
\text { Strength } \\
\left(\mathrm{KJ} / \mathrm{m}^{2}\right)\end{array}$} & \\
\hline & & & & & $\begin{array}{l}\text { Strength } \\
\text { (MPa) }\end{array}$ & $\begin{array}{l}\text { Modulus } \\
\text { (GPa) }\end{array}$ & $\begin{array}{l}\text { Strength } \\
(\mathrm{MPa})\end{array}$ & $\begin{array}{l}\text { Modulus } \\
\text { (GPa) }\end{array}$ & & \\
\hline \multirow{2}{*}{ Jute + Sisal } & & & Alkalization & & $12 \% \uparrow$ & $54.4 \% \uparrow$ & - & $21 \% \uparrow$ & $77.1 \% \uparrow$ & \\
\hline & & & Mixed (alkalization + silanization) & & $2.4 \% \uparrow$ & $9.1 \% \uparrow$ & - & $18 \% \downarrow$ & - & \\
\hline Jute & \multirow{3}{*}{$\begin{array}{c}\text { Combed } \\
\text { unidirectional } \\
\text { fibres }\end{array}$} & \multirow{3}{*}{ Epoxy } & \multirow{3}{*}{ Surface treatment } & \multirow{3}{*}{$\begin{array}{l}\text { Hand layup } \\
\text { followed by } \\
\text { compression } \\
\text { moulding method }\end{array}$} & $141.3 \% \uparrow$ & - & $4.7 \% \uparrow$ & - & $84 \% \downarrow$ & \multirow{3}{*}{ [155] } \\
\hline Sisal & & & & & $55.7 \% \uparrow$ & - & $10.8 \% \uparrow$ & - & $70.6 \% \downarrow$ & \\
\hline Banana & & & & & $182.4 \% \uparrow$ & - & $10.3 \% \uparrow$ & - & $81.4 \% \downarrow$ & \\
\hline Rice husks $(40 \% w / w)$ & Particles & Polystyrene & Compared with pure polystyrene & $\begin{array}{l}\text { Mechanical stirring } \\
\text { and single roller } \\
\text { pressing }\end{array}$ & - & $5114 \% \uparrow$ & - & - & - & [156] \\
\hline Jute fibre & \multirow{4}{*}{$\begin{array}{l}\text { Continuous } \\
\text { fibre }\end{array}$} & \multirow{4}{*}{ Polyester } & \multirow{4}{*}{$\begin{array}{l}\text { Compared with neat polyester } \\
\text { with no reinforcement }\end{array}$} & \multirow{4}{*}{$\begin{array}{l}\text { Hand lay-up } \\
\text { technique }\end{array}$} & $48 \% \uparrow$ & $100.5 \% \uparrow$ & $18.1 \% \uparrow$ & $110.6 \% \uparrow$ & $49 \% \uparrow$ & \multirow{4}{*}{ [157] } \\
\hline Bamboo fibre & & & & & $81.6 \% \uparrow$ & $99.4 \% \uparrow$ & $34.2 \% \uparrow$ & $118.7 \% \uparrow$ & $108.5 \% \uparrow$ & \\
\hline Glass-Jute fibre & & & & & $118.3 \% \uparrow$ & $121.3 \% \uparrow$ & $61.4 \% \uparrow$ & $171.5 \% \uparrow$ & $245.4 \% \uparrow$ & \\
\hline Glass-Bamboo fibre & & & & & $146.8 \% \uparrow$ & $135 \% \uparrow$ & $70.3 \% \uparrow$ & $183.7 \% \uparrow$ & $360.7 \% \uparrow$ & \\
\hline Jute fibre & $\begin{array}{l}\text { Woven } \\
\text { preforms }\end{array}$ & Epoxy & $\begin{array}{l}\text { Effect of curing temperature and } \\
\text { compared with neat matrix }\end{array}$ & $\begin{array}{l}\text { Hand lay-up } \\
\text { followed by } \\
\text { compression } \\
\text { moulding technique }\end{array}$ & $121.2 \% \uparrow$ & $50 \% \uparrow$ & $\begin{array}{l}\text { Max } 41.8 \\
\text { at } 100{ }^{\circ} \mathrm{C}\end{array}$ & - & $\begin{array}{l}\text { Max } 3.5 \mathrm{~J} \\
\text { at } 80{ }^{\circ} \mathrm{C}\end{array}$ & [139] \\
\hline Tapsi fibre (15 gms) & $\begin{array}{l}\text { Continuous } \\
\text { fibre }\end{array}$ & Epoxy & $\begin{array}{l}\text { Alkali treatment }(\mathrm{NaOH}) \text { and } \\
\text { compared with } \mathrm{KOH} \text { treatment }\end{array}$ & $\begin{array}{l}\text { Hand lay-up } \\
\text { method }\end{array}$ & $20 \% \uparrow$ & - & $3.6 \% \uparrow$ & - & - & [158] \\
\hline \multirow{4}{*}{$\begin{array}{l}\text { Elephant grass } \\
\text { fibre }(20 \%)\end{array}$} & \multirow{4}{*}{$\begin{array}{l}\text { Chopped fibre } \\
\text { strands }\end{array}$} & \multirow{4}{*}{ Polylactic acid } & $\begin{array}{l}\text { Untreated and Compared with } \\
\text { pure PLA }\end{array}$ & \multirow{4}{*}{$\begin{array}{l}\text { Injection moulding } \\
\text { technique }\end{array}$} & $21 \% \uparrow$ & $124.8 \% \uparrow$ & $17.5 \% \uparrow$ & - & $129.5 \% \uparrow$ & \multirow{4}{*}{ [159] } \\
\hline & & & $\begin{array}{c}\text { Untreated and compared with } \\
\text { Jute/PLA }\end{array}$ & & $15.5 \% \uparrow$ & - & $24.3 \% \uparrow$ & - & - & \\
\hline & & & $\begin{array}{l}\text { Untreated and compared with } \\
\text { Sisal/PLA }\end{array}$ & & $5.5 \% \uparrow$ & - & $\begin{array}{l}\text { Slightly } \\
\text { higher }\end{array}$ & - & - & \\
\hline & & & $\begin{array}{l}\text { Chemical treatment: mercerization } \\
\text { and bleaching and compared with } \\
\text { pure PLA }\end{array}$ & & $24 \% \uparrow$ & $149.6 \% \uparrow$ & $22 \% \uparrow$ & - & $\begin{array}{l}\text { Slightly } \\
\text { higher }\end{array}$ & \\
\hline
\end{tabular}


Table 4. Cont.

\begin{tabular}{|c|c|c|c|c|c|c|c|c|c|c|}
\hline \multirow{3}{*}{ Reinforcement Fibre } & \multirow{3}{*}{ Fibre Type } & \multirow{3}{*}{ Matrix } & \multirow{3}{*}{$\begin{array}{c}\text { Treatment (Fibre/Matrix } \\
\text { Modification) }\end{array}$} & \multirow{3}{*}{$\begin{array}{c}\text { Fabrication } \\
\text { Technique }\end{array}$} & \multicolumn{5}{|c|}{ Properties } & \multirow{3}{*}{ Ref. } \\
\hline & & & & & \multicolumn{2}{|c|}{ Tensile Properties } & \multicolumn{2}{|c|}{ Flexural Properties } & \multirow{2}{*}{$\begin{array}{c}\text { Impact } \\
\text { Strength } \\
\left(\mathrm{KJ} / \mathrm{m}^{2}\right)\end{array}$} & \\
\hline & & & & & $\begin{array}{l}\text { Strength } \\
\text { (MPa) }\end{array}$ & $\begin{array}{l}\text { Modulus } \\
\text { (GPa) }\end{array}$ & $\begin{array}{l}\text { Strength } \\
\text { (MPa) }\end{array}$ & $\begin{array}{l}\text { Modulus } \\
\text { (GPa) }\end{array}$ & & \\
\hline & & & $\begin{array}{l}\text { Chemical treatment: mercerization } \\
\text { and bleaching and compared with } \\
\text { Jute/PLA }\end{array}$ & & $18.14 \% \uparrow$ & - & $\begin{array}{l}\text { Slightly } \\
\text { higher }\end{array}$ & - & - & \\
\hline & & & $\begin{array}{l}\text { Chemical treatment: mercerization } \\
\text { and bleaching and compared with } \\
\text { Sisal/PLA }\end{array}$ & & $\begin{array}{l}\text { Slightly } \\
\text { higher }\end{array}$ & - & $4 \% \uparrow$ & - & - & \\
\hline & & & $\begin{array}{c}\text { Chemical treatment: mercerization } \\
\text { and bleaching and compared with } \\
\text { untreated grass/PLA }\end{array}$ & & - & - & $28 \% \uparrow$ & - & - & \\
\hline Tossa jute fibre & $\begin{array}{l}\text { Commercially } \\
\text { available fibre }\end{array}$ & Epoxy & Fibre corona treatment & $\begin{array}{l}\text { Compression } \\
\text { moulding }\end{array}$ & - & - & $30 \% \uparrow$ & - & - & {$[160]$} \\
\hline Flax fibre & $\begin{array}{l}\text { Randomly } \\
\text { oriented fibre }\end{array}$ & Polyester & Fibre plasma treatment & $\begin{array}{l}\text { Compression } \\
\text { moulding }\end{array}$ & - & $16.5 \% \uparrow$ & - & - & - & {$[161]$} \\
\hline kraft fibre & $\begin{array}{c}\text { Randomly } \\
\text { oriented fibre }\end{array}$ & Polypropylene & Fibre-beating treatment & Extrusion moulding & $10 \% \uparrow$ & - & - & - & - & {$[162]$} \\
\hline Jute fibre & $\begin{array}{l}\text { Chopped fibre } \\
\text { strands }\end{array}$ & Vinyl ester & Fibre alkaline treatment & $\begin{array}{c}\text { Compression } \\
\text { moulding }\end{array}$ & - & - & $35 \% \uparrow$ & $23 \% \uparrow$ & - & {$[163]$} \\
\hline Hemp fibre & $\begin{array}{c}\text { Randomly } \\
\text { oriented fibre }\end{array}$ & Polypropylene & Fibre silane treatment & $\begin{array}{l}\text { Compression } \\
\text { moulding }\end{array}$ & $4 \% \uparrow$ & - & $2 \% \uparrow$ & - & - & {$[164]$} \\
\hline Flax fibre & $\begin{array}{c}\text { Randomly } \\
\text { oriented fibre }\end{array}$ & Polypropylene & Fibre acetylation treatment & Injection moulding & $35 \% \uparrow$ & - & $35 \% \uparrow$ & - & - & {$[165]$} \\
\hline Sisal fibre & $\begin{array}{l}\text { Chopped fibre } \\
\text { strands }\end{array}$ & Polystyrene & Fibre benzoylation treatment & $\begin{array}{l}\text { Compression } \\
\text { moulding }\end{array}$ & $91 \% \uparrow$ & - & - & - & - & {$[166]$} \\
\hline Jute fibre & $\begin{array}{c}\text { Randomly } \\
\text { oriented fibre }\end{array}$ & Polypropylene & $\begin{array}{l}\text { Fibre treated by a maleated } \\
\text { coupling agent }\end{array}$ & $\begin{array}{l}\text { Compression } \\
\text { moulding }\end{array}$ & $15.4 \% \uparrow$ & $22.4 \% \uparrow$ & - & - & - & {$[167]$} \\
\hline \multirow{2}{*}{ Hemp fibre } & \multirow{2}{*}{$\begin{array}{l}\text { Randomly } \\
\text { oriented fibre }\end{array}$} & \multirow{2}{*}{ Polypropylene } & Fibre fungal treatment & \multirow{2}{*}{ Injection moulding } & $22 \% \uparrow$ & - & - & - & - & \multirow{2}{*}[168]{} \\
\hline & & & Fibre fungal and alkaline treatment & & $22 \% \uparrow$ & - & - & - & - & \\
\hline
\end{tabular}




\section{Factors Affecting the Mechanical Properties of Biocomposites}

Many factors, such as type of polymer matrix and the types associated fibres; the fibre's origin, processing and forms; the fibre dispersion; the distribution in the matrix; the orientation; the fibrematrix interfacial interaction; and the techniques used in the composite's fabrication, directly affect the mechanical properties of the biocomposites [169-174]. Besides, as the strength of reinforcement fibre is higher than that of the matrix material, the strength of a biocomposite is more dependent on the fibre rather than the matrix [175-178]. Therefore, the strength of a biocomposite can be enhanced by increasing the amount of fibre in the composite structure to a certain extent $[179,180]$. On the other hand, when the amount of fibre exceeds the limit, the load transferred through the interface reduces, and the amount of porosity in the composite increases, which results in a greater reduction in the strength and stiffness of the composite material [179]. Moreover, with the increment of fibre content into the composite structure, the water uptake of the composite increases, which very often degrades the mechanical properties of the composite material [181-183]. Along with the amount of fibre content, the length of the reinforcement fibre plays an important role in the mechanical properties of the composite [24]. The higher the aspect ratio of the fibre, the better its load-bearing capacity [184]. However, it should be noted that a very long fibre can become entangled, resulting in non-uniform distribution of the fibre and the reinforcement [185]. The matrix is also an important constituent of the composite that protects the fibre's surface from externally applied forces. The polymer matrix transfers the forces externally applied to the composite to the reinforcement materials, and thus enhances the composite's longevity [186]. With the increased interfacial interaction between the fibres and the matrices, the biocomposites become stronger. As the applied load is transferred from the matrix to the reinforcement materials, the interfacial bonding is all important and is the true indicator of the composite properties [170,187]. To increase the interfacial bonding between the fibre and the matrix, it is necessary to improve the hydrophobicity of the fibres, the interfacial bonding between matrix and fibre and the roughness [188]. Bonding between fibre and matrix is usually enhanced by a few mechanisms, such as mechanical interlocking, chemical bonding, electrostatic bonding and inter-diffusion bonding [189-195]. Besides, the other factors such as temperature, pressure, time and resin viscosity seriously affect the properties of the biocomposites [139,196-198].

\section{Biodegradability of the Biocomposites}

The natural fibre reinforced composites (NFRCs) show excellent biodegradability compared to the synthetic fibre-based materials and composites, which is one of the major aspects of fabricating NFRCs $[199,200]$. In general, the biodegradable materials are degraded and converted into $\mathrm{CO}_{2}$, $\mathrm{H}_{2} \mathrm{O}$, hydrocarbons, methane and biomass under the aerobic or anaerobic conditions, due to the chemical or biological reactions [200-202]. In most of the cases, the material biodegradability is tested by the soil burial method (ASTM D5988/D5338 or ISO 14855) and the results are expressed by the weight loss (\%) [199,203-206]. Different factors such as the molecular weight, chemical structure, glass transition temperature $(\mathrm{Tg})$, melting temperature $(\mathrm{Tm})$, mechanical behaviour, crystallinity and crystal structure potentially impact the NFRC's biodegradability $[199,203]$. For example, a polymer with high crystallinity and a high melting temperature shows lower biodegradability-i.e., lower weight loss $(\%)[200,203]$. A study over a 3-month period revealed that the biodegradability of the lyocell incorporated polyester composites is higher $(75 \%)$ than that of the pure polyester, which might be due to the presence of natural lyocell fibre [207]. In another study, the biodegradability test of the kenaf/PLA composites using the garbage-processing machine showed the weight loss of $38 \%$ within one-month of composting [208]. Recently, Wu et al. found that the use of $40 \mathrm{wt} . \%$ palm fibre (PF) as a reinforcement with the polyhydroxyalkanoate (PHA) matrix increased the rate of biodegradation $(\sim 90 \%)$ compared to pure PHA ( 20\%) [209]. Similarly, the study of Mittal and Chaudhary revealed that the biodegradability of the epoxy resin increased while using the nature-based pineapple and coconut fibres as reinforcements with the epoxy resin. Pure epoxy exhibited only $10 \%$ weight loss, while the natural fibre reinforced composites showed $60 \%$ to $80 \%$ weight loss [210]. The authors concluded that 
upon burial of the composites into the soil, both the water molecules and microorganisms penetrated the polymeric backbone, which eventually degraded the higher cellulose contents of the natural pineapple and coir fibres, resulting in the higher weight losses (\%) of the composite materials compared to the pure synthetic materials [210]. The biodegradability of some of the NFRCs reported in the literature is shown in Table 5.

Table 5. Biodegradability of the natural fibre reinforced composites (NFRCs).

\begin{tabular}{|c|c|c|c|}
\hline NFRCs Composition (wt.\%) & $\begin{array}{l}\text { Biodegradability Test } \\
\text { Conditions }\end{array}$ & Outcomes & Ref. \\
\hline $\begin{array}{l}\text { Polybutylene succinate } \\
\text { (PBS)/Sugarcane rind fibre } \\
\text { (SRF) (95:5) }\end{array}$ & Soil burial test (100 days) & $\begin{array}{l}\text { Maximum weight loss }(\sim 20 \%) \\
\text { was found with the PBS/SRF } \\
\text { composites than that of pure } \\
\text { PBS }(\sim 5 \%) \text {. }\end{array}$ & [211] \\
\hline $\begin{array}{c}\text { Polybutylene } \\
\text { adipate-co-terephthalate } \\
\text { (PBAT)/Distillers dried grains } \\
\text { with soluble (DDGS) (70:30) }\end{array}$ & Compost (ASTM D5338) & $\begin{array}{l}\text { The PBAT/DDGS composite } \\
\text { showed higher biodegradability } \\
(\sim 98 \%) \text { compared to the neat } \\
\text { PBAT }(\sim 92 \%) \text {. }\end{array}$ & [204] \\
\hline $\begin{array}{l}\text { Polylactic acid (PLA)/Maple } \\
\text { wood fibre (70:30) }\end{array}$ & Compost (ISO-14855) & $\begin{array}{l}\text { Acetyl treatment of the maple } \\
\text { wood fibres increased their } \\
\text { porosity that enhanced the } \\
\text { hydrolytic degradation of PLA. }\end{array}$ & [205] \\
\hline $\begin{array}{l}\text { Polypropylene (PP)/Bamboo } \\
(50: 50)\end{array}$ & $\begin{array}{l}\text { Soil burial test } \\
\text { (ASTM D5988) }\end{array}$ & $\begin{array}{l}15 \% \text { biodegradability was } \\
\text { observed within } 130 \text { days. }\end{array}$ & [206] \\
\hline $\begin{array}{c}\text { Polyethylene sebacate } \\
\text { (PES)/Acylated cellulose fibre } \\
(85: 15)\end{array}$ & Compost (ASTM D5338) & $\begin{array}{l}\text { Within } 30 \text { days, the } \\
\text { biocomposite products showed } \\
100 \% \text { biodegradability. }\end{array}$ & [212] \\
\hline $\begin{array}{l}\text { Polylactic acid (PLA)/Kenaf } \\
\text { (80:20) and Polylactic acid } \\
\text { (PLA)/Rice husk (80:20) }\end{array}$ & Soil Burial Test (90 days) & $\begin{array}{l}\text { The PLA/Kenaf and PLA/Rice } \\
\text { husk composites showed } \\
\text { 3-times and 2-times higher } \\
\text { weight loss (\%), respectively } \\
\text { compared to the neat PLA. }\end{array}$ & [213] \\
\hline $\begin{array}{c}\text { Polylactic acid (PLA)/Soy } \\
\text { straw (70:30) }\end{array}$ & Compost (ASTM D5338) & $\begin{array}{l}\text { In } 60 \text { days, the PLA/Soy straw } \\
\text { exhibited } 90 \% \text { degradation while } \\
\text { the pure PLA showed } 50 \% \text {. }\end{array}$ & [214] \\
\hline
\end{tabular}

\section{Challenges Associated with the Biocomposites and Probable Fibre Modifications}

The development, production and applications of the plant-based natural fibre reinforced composite materials (NFRCs) are increasing regularly due to their outstanding properties. However, worldwide researchers are facing a number of challenges relating to the development of $100 \%$ green biocomposites where both the polymer matrices and reinforcing materials come from natural and renewable resources. Additionally, poor interfacial bonding between the polymer matrix and the reinforcing material, due to the hydrophilic nature of plant-based natural fibres and hydrophobicity of the polymer matrices, is another point of consideration while fabricating the cellulose-based NFRCs [170,215]. Due to the low interfacial interaction, the NFRCs cannot ensure the desired mechanical properties, although they provide the most pursued biodegradability properties, and are significantly cheaper as compared to the high-performance synthetic fibres, such as carbon fibre $[170,216]$. On top of that, low thermal stability, high moisture absorbency and low wettability of cellulosic natural fibres are some other remarkable challenges in the production of the NFRCs [217]. Moreover, it is not always possible to ensure the similar properties among the natural fibres, as their properties vary based on the changes in weather, season, cultivation conditions and production processing [218,219]. Hence, necessary modifications of the reinforcement fibres must be taken into consideration before processing the improvement of interfacial bonding by enhancing the wettability of the fibres and controlling the moisture absorption of the fibres or matrix [220]. The fibre and polymer matrix 
interfacial bonding can be improved by physical and chemical fibre modification techniques. Interfacial bonding between the reinforcement fibres and the polymer matrix can be improved by applying some of the fibre modification techniques-both physical and chemical.

The physical modification of a cellulosic natural fibre refers to the changing of the surface properties such as surface energy, polarity, surface area, cleanliness and wettability. Corona treatment [160,221-223], plasma treatment [224-226], ultraviolet (UV) treatment [227-230], fibre beating [231-233] and heat treatment [234] are some of the remarkable techniques for physical modifications of fibre without hampering the chemical structure of fibres [235-237]. In the case of the corona, for plasma and UV treatment, fibre surface energy is changed using a high voltage at low temperature and atmospheric pressure [160]. Consequently, wettability and fibre-matrix interfacial bonding is improved, which enhances the composite strength. Active surface area is enlarged by the process of fibre beating which allows a good interfacial interaction between fibre and matrix [162]. The heat treatment process removes the non-cellulosic components of plant fibres, such as pectin, lignin and hemicellulose, along with other dirt from the fibres, and allows the cleaned fibre to react with the matrix more actively [238]. The chemical treatment is mostly carried out to deteriorate the inherent hydrophilic nature of the natural fibres, which eventually assists in enhancing the interfacial bonding between the fibres and the matrix $[239,240]$. Over the past few decades, very intensive research work has continued to better understand the chemical treatment behaviour of cellulosic fibres. Presently, mercerisation [241-245], acetylation [246-249], peroxide treatment [250-252], permanganate treatment [253-257], silanization [258-264], acrylation [265-269], acrylonitrile grafting [270-272] and latex coating [273-275] are the chemical modification techniques most used to improve the reactivity of the natural fibres with the polymeric matrix materials. The hydrophilicity reduction of the cellulosic fibres by eliminating the proportions of hydroxyl groups $(-\mathrm{OH})$ is the ultimate objective of the abovementioned chemical modification techniques [24]. While, due to the reduction in the number of $-\mathrm{OH}$ groups, the moisture absorbency of the fibres is reduced, the non-cellulosic components and dirt are removed as the by-products of the chemical reaction, which allows an improved load transfer capacity through the fibre-matrix interface. Figure 6 illustrates the often used chemical modification techniques associated with the cellulosic fibres [276].

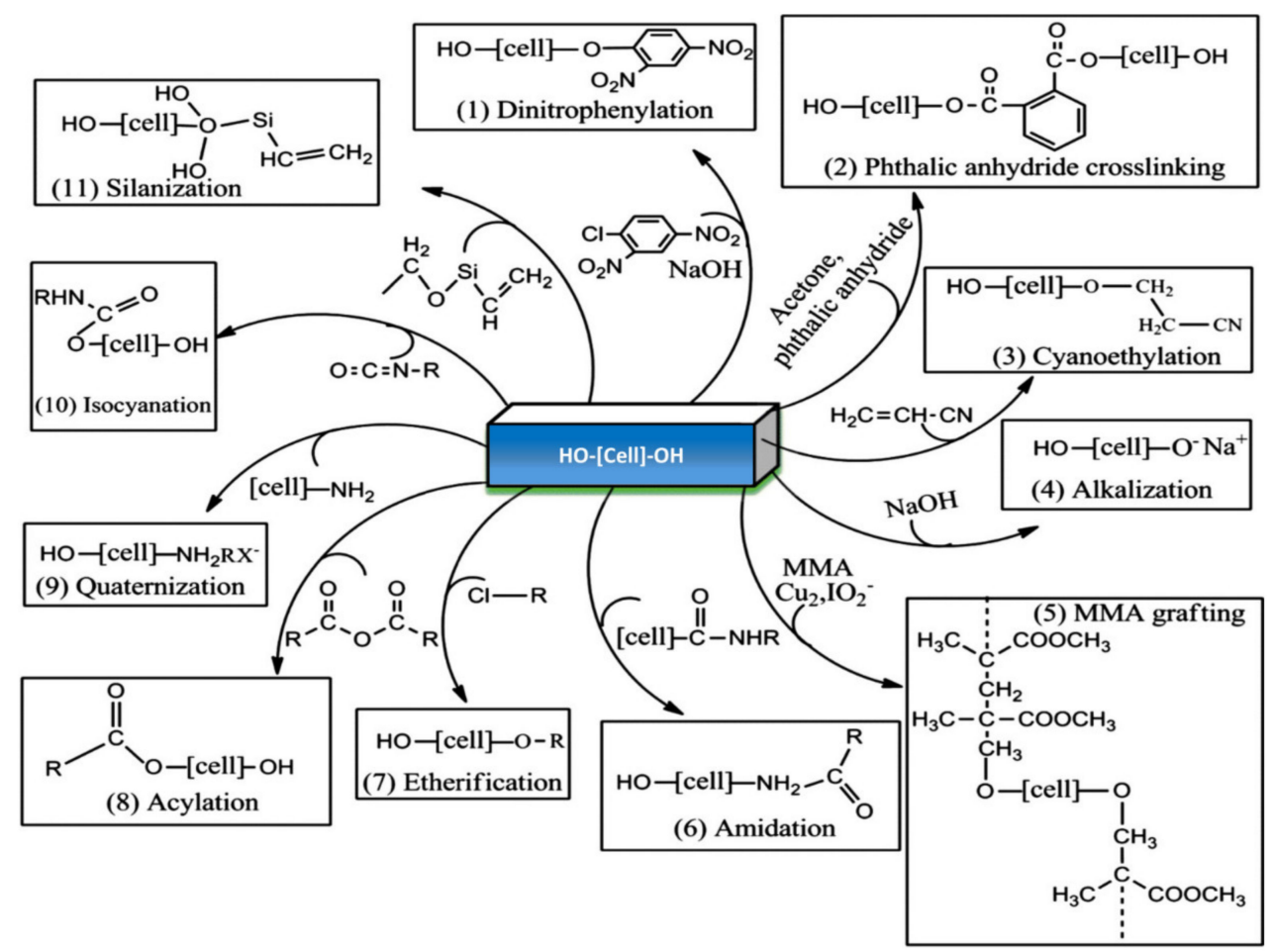

Figure 6. Chemical modifications of the plant-based fibres for biocomposites (Reprinted with permission from [276]; Copyright 2015 Elsevier). 


\section{Potential Application Areas of Biocomposites}

Many countries across the world have imposed restrictions for industries in the use of oil-based raw materials while fabricating different products $[277,278]$. For example, in the year of 2005, the European Commission (EU) executed "European Guideline 2000/53/EG," which ensured the utilization of 85\% recyclable materials while fabricating the automobile parts [21]. This amount was further augmented to $95 \%$ in the year of 2015 [22]. Light weight but greater mechanical strength; lower manufacturing costs; resistance to fatigue and corrosion; and greater availability, renewability and biodegradability than synthetic fibres, are some of the major reasons for this increased demand of NFRCs [279-282]. The potential application areas have been increasing dramatically over the last few decades, including but not limited to the automobile, packaging, military, sports, medical, building and constructions sectors [279-281]. Among these, the applications of NFRCs are the most prevalent in the automobile industry, due to the requirements for eco-friendly lightweight materials and associated costs $[9,13,55]$. Henry Ford fabricated the first NFRCs using hemp fibres in the year of 1940 [22]. Later, several car manufacturers reported the fabrication of car bodies and other associated parts from NFRCs in the 1950s and in between 1990 and 1996, which gradually increased and gained popularity [13,22], and in recent years from 2014 to 2019, an increase of $11 \%$ in production was reported [283].

The natural fibre-based composite market is expected to grow to $\$ 531.3$ million US in the year 2019 from $\$ 289.3$ million in 2010 with $28 \%$ of market shares occupied by natural fibres [283,284]. Table 6 shows the main producers and the annual production of some plant-based natural fibres commonly used in NFRCs.

Table 6. Producers and annual production of most widely used plant-based natural fibres [56-60].

\begin{tabular}{|c|c|c|c|}
\hline Fibre & Producer & $\begin{array}{l}\text { Production Amount } \\
\quad\left(\times 10^{3} \text { ton }\right)\end{array}$ & Price (US\$/ton) ${ }^{a}$ \\
\hline Abaca & Philippines (85\%), Ecuador & 70 & 345 \\
\hline Bagasse & Brazil, China, India, Thailand, Australia, USA & 75,000 & $3.5-11.8(7.65)$ \\
\hline Coir & $\begin{array}{l}\text { India, Sri Lanka, Thailand, Vietnam, } \\
\text { Philippines, Indonesia, Brazil }\end{array}$ & 1200 & $200-500(350)$ \\
\hline Cotton & $\begin{array}{c}\text { China, Brazil, India, Pakistan, USA, } \\
\text { Uzbekistan, Turkey }\end{array}$ & 25,000 & $1500-4200(2850)$ \\
\hline Flax & $\begin{array}{l}\text { France, Belgium, Netherland, Poland, Russian } \\
\text { Federation, China }\end{array}$ & 830 & $2100-4200(3150)$ \\
\hline Jute & India $(60 \%)$, Bangladesh, Myanmar, Nepal & 3450 & $400-1500(950)$ \\
\hline Kapok & $\begin{array}{l}\text { Philippine, Malaysia, China, South America, } \\
\text { Indonesia, Thailand }\end{array}$ & 101 & 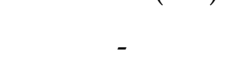 \\
\hline Kenaf & $\begin{array}{c}\text { India }(45 \%), \text { China, Malaysia, USA, Mexico, } \\
\text { Thailand, Vietnam }\end{array}$ & 970 & $300-500(400)$ \\
\hline Bamboo & $\begin{array}{c}\text { China, Japan, India, Chile, Ecuador, Indonesia, } \\
\text { Myanmar, Nigeria, Sri Lanka, } \\
\text { Philippines, Pakistan }\end{array}$ & 30,000 & 500 \\
\hline Hemp & China (80\%), Chile, France, Germany, UK & 214 & 1000-2100 (1550) \\
\hline Ramie & China, Brazil, Lao PDR, Philippines, India & 280 & 2000 \\
\hline Sisal & $\begin{array}{l}\text { Brazil ( } 40 \% \text { ), Kenya, Tanzania, China, Cuba, } \\
\text { Haiti, Madagascar, Mexico, Sri Lanka, India }\end{array}$ & 378 & $600-700(650)$ \\
\hline Banana & $\begin{array}{c}\text { India }(22 \%), \text { China, Philippines, Ecuador } \\
\text { and Brazil }\end{array}$ & 134,000 & 890 \\
\hline Pineapple & $\begin{array}{c}\text { Costa Rica, Philippines, Taiwan, Brazil, Hawaii, } \\
\text { India, Indonesia }\end{array}$ & 1318 & $360-550(455)$ \\
\hline
\end{tabular}

It has been reported that application of NFRCs would result in the reduction of nearly $20 \%$ of manufacturing costs and 30\% of automobile weight which would also help in reducing the fuel consumption $[9,55]$. The European car manufacturers are making serious attempts at incorporating the NFRCs in various portions of cars, such as car seats, backrests, front and back door liners and in door-trim panels [285]. The German car manufacturers are currently focusing on the fabrication of 
exterior body parts for cars and trucks with bio-based composites $[25,55,286]$. It has been reported that in the year of 2004 the BMW group used 10 kilotonnes of renewable natural fibres while producing the NFRCs for their cars [287]. Table 7 shows the utilisation of different plant-based natural fibres in various parts of the automobiles [9,55,288-291]. It has been reported that in the year of 2012, the European automobile industry has used $38 \%$ wood, $25 \%$ cotton, $19 \%$ flax, $8 \%$ kenaf, $5 \%$ hemp and $7 \%$ other natural fibres such as jute, coir, sisal and abaca to fabricate the natural fibre reinforced composites (NFRCs) to use in this particular industry [290]. Hence, it can be predicted that in near future the NFRCs would be used in a higher proportion in the automobile industry.

Table 7. Applications of the plant-based natural fibre reinforced composites (NFRCs) in the automobile industry $[9,55,288-291]$.

\begin{tabular}{ccc}
\hline Car Manufacturer & Model & Application Areas \\
\hline Rover & 2000 and others & Insulations, rear storage panel \\
Audi & A2, A3, A4, A6, A8 & Seatback, side and back door panel, spare-tire lining, \\
boot-liner
\end{tabular}

In addition to the automobile industry, the biocomposites fabricated from the cellulosic natural fibres are extensively used in geotextiles, medicine, sports, construction and packaging (Table 8). After the automobile industry, the building and construction industries are the second-largest consumers of NFRCs owing to the demand for the eco-friendly green buildings, and load-bearing and non-load bearing capabilities of NFRCs [292,293]. Roofs, windows, panels and doors are built using the NFRCs [293]; for example, the composites fabricated from sisal fibres can be used in producing tanks, pipes and even roofing materials that could replace asbestos [294,295].

Table 8. Applications of cellulosic fibres in different industries [296-299].

\begin{tabular}{cc}
\hline Fibres & Industrial Applications \\
\hline Hemp & Textiles, geotextiles, paper and packaging, electrical, furniture, cordage, construction items, \\
producing banknotes and manufacturing pipes
\end{tabular}




\section{Conclusions}

Plant-based natural fibre reinforced composites (NFRCs) have some excellent properties, such as biodegradability, biocompatibility and renewability. Hence, it is more appropriate to use the NFRCs instead of the petroleum-based synthetic materials, which are neither eco-friendly nor obtained from the renewable sources. This review thoroughly illustrated the required properties of the cellulosic fibres and the polymeric matrices for the fabrication of the NFRCs. Additionally, the mechanical properties, challenges and potential application areas of the NFRCs have been discussed. Although it is evident that the plant fibres are not free from some drawbacks, such as possessing higher moisture retention, these can be overcome by applying various physical and chemical modifications. Moreover, it is difficult to produce $100 \%$ green biocomposites wherein both the polymeric matrix and the reinforcing material are derived from the natural and renewable sources. Therefore, more focus is required to commercially develop pure green biocomposites to support sustainability. It is necessary to find new sources of natural biopolymers and fibres which can be used as both the polymer matrices and the reinforcing materials. Research also needs to find how can we utilize the abundantly available short and non-spinnable natural fibre wastes, either as reinforcing materials or as polymer matrices to fabricate fully green biocomposites.

Author Contributions: M.S., original idea, conceptualization, methodology, formal analysis, investigation, resources, writing - original draft preparation and reference management; M.A.A.F., formal analysis, resources, methodology and writing - original draft preparation; K.B., conceptualization, validation, writing-review and editing, visualization and supervision; M.N., validation, writing-review and editing, visualization and supervision. All authors have read and agreed to the published version of the manuscript.

Funding: This research received no external funding.

Conflicts of Interest: The authors declare no conflict of interest.

\section{Abbreviations}

$\begin{array}{ll}\text { NFRCs } & \text { Natural Fibre Reinforced Composites } \\ \text { PLA } & \text { Polylactic Acid } \\ \text { PE } & \text { Polyethylene } \\ \text { PP } & \text { Polypropylene } \\ \text { PAN } & \text { Polyacrylonitrile } \\ \text { PC } & \text { Polycarbonates } \\ \text { RTM } & \text { Resin Transfer Moulding } \\ \text { UD } & \text { Unidirectional } \\ \text { PF } & \text { Palm fibre } \\ \text { PBS } & \text { Polybutylene succinate } \\ \text { PBAT } & \text { Polybutylene adipate-co-terephthalate } \\ \text { PES } & \text { Polyethylene sebacate } \\ \text { PS } & \text { Polystyrene } \\ \text { PMMA } & \text { Polymethyl methacrylate } \\ \text { PVC } & \text { Polyvinyl chloride } \\ \text { PVA } & \text { Polyvinyl acetate } \\ \text { PET } & \text { Polyethylene terephthalate } \\ \text { RFI } & \text { Resin Film Infusion } \\ \text { VaRTM } & \text { Vacuum-assisted resin transfer moulding } \\ \text { CP } & \text { Cross ply } \\ \text { PHA } & \text { Polyhydroxyalkanoate } \\ \text { SRF } & \text { Sugarcane rind fibre } \\ \text { DDGS } & \text { Distillers dried grains with soluble } \\ \end{array}$




\section{References}

1. Faruque, M.A.A.; Remadevi, R.; Razal, J.; Wang, X.; Naebe, M. Investigation on structure and characteristics of alpaca-based wet-spun polyacrylonitrile composite fibres by utilizing natural textile waste. J. Appl. Polym. Sci. 2020, 137, 48370. [CrossRef]

2. Faruque, A.; Remadevi, R.; Wang, X.; Naebe, M. Preparation and characterisation of mechanically milled particles from waste alpaca fibres. Powder Technol. 2019, 342, 848-855. [CrossRef]

3. Cai, Z.; Remadevi, R.; Al Faruque, A.; Setty, M.; Fan, L.; Haque, A.N.M.A.; Naebe, M. Fabrication of a cost-effective lemongrass (Cymbopogon citratus) membrane with antibacterial activity for dye removal. RSC Adv. 2019, 9, 34076-34085. [CrossRef]

4. Faruque, M.A.A.; Remadevi, R.; Razal, J.; Wang, X.; Naebe, M. Impact of the wet spinning parameters on the alpaca-based polyacrylonitrile composite fibres: Morphology and enhanced mechanical properties study. J. Appl. Polym. Sci. 2020, 137, 49264. [CrossRef]

5. Safri, S.N.A.; Sultan, M.T.H.; Jawaid, M.; Jayakrishna, K. Impact behaviour of hybrid composites for structural applications: A review. Compos. Part B Eng. 2018, 133, 112-121. [CrossRef]

6. Khan, S.; Malik, A. Environmental and Health Effects of Textile Industry Wastewater. In Environmental Deterioration and Human Health; Springer Science and Business Media LLC: New York, NY, USA, 2013; pp. 55-71.

7. Mishra, S.; Charan Rath, C.; Das, A.P. Marine microfibre pollution: A review on present status and future challenges. Mar. Pollut. Bull. 2019, 140, 188-197. [CrossRef]

8. Katip, A.; İnce, Z. Green chemistry applications in textile industry. Sci. Ecol. Eng. Res. Glob. World 2018, 31, 384.

9. Mohammed, L.; Ansari, M.M.N.; Pua, G.; Jawaid, M.; Islam, M.S. A review on natural fibre reinforced polymer composite and its applications. Int. J. Polym. Sci. 2015, 2015. [CrossRef]

10. Ramamoorthy, S.K.; Skrifvars, M.; Persson, A. A review of natural fibres used in biocomposites: Plant, animal and regenerated cellulose fibres. Polym. Rev. 2015, 55, 107-162. [CrossRef]

11. Väisänen, T.; Das, O.; Tomppo, L. A review on new bio-based constituents for natural fibre-polymer composites. J. Clean. Prod. 2017, 149, 582-596. [CrossRef]

12. Remadevi, R.; Al Faruque, A.; Zhang, J.; Naebe, M. Electrically conductive honeycomb structured graphene composites from natural protein fibre waste. Mater. Lett. 2020, 264, 127311. [CrossRef]

13. Pico, D.; Steinmann, W. Synthetic fibres for composite applications. In Fibrous and Textile Materials for Composite Applications; Springer: Berlin/Heidelberg, Germany, 2016; pp. 135-170.

14. Pickering, S.J. Recycling technologies for thermoset composite materials-Current status. Compos. Part A Appl. Sci. Manuf. 2006, 37, 1206-1215. [CrossRef]

15. Henshaw, J.M.; Han, W.; Owens, A.D. An Overview of Recycling Issues for Composite Materials. J. Thermoplast. Compos. Mater. 1996, 9, 4-20. [CrossRef]

16. Huda, M.S.; Drzal, L.T.; Mohanty, A.K.; Misra, M. Chopped glass and recycled newspaper as reinforcement fibers in injection molded poly(lactic acid) (PLA) composites: A comparative study. Compos. Sci. Technol. 2006, 66, 1813-1824. [CrossRef]

17. Mitra, B.C. Environment Friendly Composite Materials: Biocomposites and Green Composites. Def. Sci. J. 2014, 64, 244-261. [CrossRef]

18. Maghsoudi-Ganjeh, M.; Lin, L.; Wang, X.; Zeng, X. Bioinspired design of hybrid composite materials. Int. J. Smart Nano Mater. 2018, 10, 90-105. [CrossRef]

19. Subramani, N.; Murali, J.G.; Suresh, P.; Sankar, V.V.A. Review on hybrid composite materials and its applications. Int. Res. J. Eng. Technol. 2017, 4, 1921.

20. Kanitkar, Y.M.; Kulkarni, A.P.; Wangikar, K.S. Characterization of Glass Hybrid composite: A Review. Mater. Today Proc. 2017, 4, 9627-9630. [CrossRef]

21. Peças, P.; Carvalho, H.; Salman, H.; Leite, M. Natural Fibre Composites and Their Applications: A Review. J. Compos. Sci. 2018, 2, 66. [CrossRef]

22. Witayakran, S.; Smitthipong, W.; Wangpradid, R.; Chollakup, R.; Clouston, P.L. Natural Fibre Composites: Review of Recent Automotive Trends; Elsevier: London, UK, 2017; pp. 166-174.

23. Toldy, A.; Niedermann, P.; Rapi, Z.; Szolnoki, B. Flame retardancy of glucofuranoside based bioepoxy and carbon fibre reinforced composites made thereof. Polym. Degrad. Stab. 2017, 142, 62-68. [CrossRef] 
24. Pickering, K.L.; Efendy, M.A.; Le, T.M. A review of recent developments in natural fibre composites and their mechanical performance. Compos. Part A Appl. Sci. Manuf. 2016, 83, 98-112. [CrossRef]

25. Mohanty, A.K.; Misra, M.; Drzal, L.T. Natural Fibres, Biopolymers, and Biocomposites; CRC Press: Boca Raton, FL, USA, 2005.

26. Lampke, T.; Mishra, S.; Bismarck, A. Plant fibres as reinforcement for green composites. In Natural Fibres, Biopolymers, and Biocomposites; CRC Press: Boca Raton, FL, USA, 2005; pp. 52-128.

27. Khan, M.Z.; Srivastava, S.K.; Gupta, M. Tensile and flexural properties of natural fiber reinforced polymer composites: A review. J. Reinf. Plast. Compos. 2018, 37, 1435-1455. [CrossRef]

28. Shinoj, S.; Visvanathan, R.; Panigrahi, S.; Kochubabu, M. Oil palm fibre (OPF) and its composites: A review. Ind. Crops Prod. 2011, 33, 7-22. [CrossRef]

29. La Mantia, F.; Morreale, M. Green composites: A brief review. Compos. Part A Appl. Sci. Manuf. 2011, 42, 579-588. [CrossRef]

30. Mukherjee, T.; Kao, N. PLA Based Biopolymer Reinforced with Natural Fibre: A Review. J. Polym. Environ. 2011, 19, 714-725. [CrossRef]

31. Shah, A.U.R.; Prabhakar, M.N.; Song, J.-I. Current advances in the fire retardancy of natural fibre and bio-based composites-A review. Int. J. Precis. Eng. Manuf. Technol. 2017, 4, 247-262. [CrossRef]

32. Wang, X.; Wang, D.-Y. Fire-retardant polylactic acid-based materials. In Novel Fire Retardant Polymers and Composite Materials; Elsevier: Amsterdam, The Netherlands, 2017; pp. 93-116.

33. Sanjay, M.; Madhu, P.; Jawaid, M.; Senthamaraikannan, P.; Senthil, S.; Pradeep, S. Characterization and properties of natural fiber polymer composites: A comprehensive review. J. Clean. Prod. 2018, 172, 566-581. [CrossRef]

34. Sanjay, M.; Siengchin, S.; Parameswaranpillai, J.; Jawaid, M.; Pruncu, C.I.; Khan, A. A comprehensive review of techniques for natural fibers as reinforcement in composites: Preparation, processing and characterization. Carbohydr. Polym. 2019, 207, 108-121. [CrossRef]

35. Kerni, L.; Singh, S.; Patnaik, A.; Kumar, N. A review on natural fiber reinforced composites. Mater. Today Proc. 2020, 28, 1616-1621. [CrossRef]

36. Li, M.; Pu, Y.; Thomas, V.M.; Yoo, C.G.; Ozcan, S.; Deng, Y.; Nelson, K.; Ragauskas, A.J. Recent advancements of plant-based natural fiber-reinforced composites and their applications. Compos. Part B Eng. 2020, 200, 108254. [CrossRef]

37. Scaffaro, R.; Lopresti, F.; Botta, L. PLA based biocomposites reinforced with Posidonia oceanica leaves. Compos. Part B Eng. 2018, 139, 1-11. [CrossRef]

38. Wang, G.; Zhang, D.; Wan, G.; Li, B.; Zhao, G. Glass fibre reinforced PLA composite with enhanced mechanical properties, thermal behavior, and foaming ability. Polymer 2019, 181, 121803. [CrossRef]

39. Koronis, G.; Silva, A.; Fontul, M. Green composites; A review of adequate materials for automotive applications. Compos. Part B Eng. 2013, 39, 120-127. [CrossRef]

40. Dinesh, S.; Kumaran, P.; Mohanamurugan, S.; Vijay, R.; Singaravelu, D.L.; Vinod, A.; Sanjay, M.R.; Siengchin, S.; Bhat, K.S. Influence of wood dust fillers on the mechanical, thermal, water absorption and biodegradation characteristics of jute fibre epoxy composites. J. Polym. Res. 2020, 27, 9. [CrossRef]

41. Barbero, E.J. Introduction to Composite Materials Design, 3rd ed.; CRC Press: London, UK, 2017.

42. Akovali, G. Handbook of Composite Fabrication; Rapra Technology Limited: Shorpshire, UK, 2001; pp. 3-181.

43. Bunsell, A.; Renard, J. Fundamentals of Fibre Reinforced Composite Materials. Ser. Mater. Sci. Eng. 2005. [CrossRef]

44. Balasubramanian, K.; Sultan, M.T.; Rajeswari, N. Manufacturing Techniques of Composites for Aerospace Applications; Elsevier: Amsterdam, The Netherlands, 2018; pp. 55-67.

45. Boisse, P. Advances in Composites Manufacturing and Process Design; Woodhead Publishing: Cambridge, UK, 2015.

46. Chung, D.D.L. Carbon Composites: Composites with Carbon Fibres, Nanofibres, and Nanotubes; ButterworthHeinemann: Oxford, UK, 2016.

47. Nurazzi, M.; Laila, D. A Review: Fibres, Polymer Matrices and Composites science \& technology. Polym. Matrices Compos. 2017, 25, 1085-1102.

48. Tridico, S.R. Natural animal textile fibres: Structure, characteristics and identification. In Woodhead Publishing Series in Textiles; Houck, M., Ed.; Woodhead Publishing: Cambridge, UK, 2009; pp. 27-67. 
49. Naebe, M.; McGregor, B.A.; Swan, P.; Tester, D. Associations between the physiological basis of fabric-evoked prickle, fibre and yarn characteristics and the Wool ComfortMeter value. Text. Res. J. 2015, 85, 1122-1130. [CrossRef]

50. Muthuraj, R.; Misra, M.; Mohanty, A.K. Studies on mechanical, thermal, and morphological characteristics of biocomposites from biodegradable polymer blends and natural fibres. In Biocomposites; Elsevier: Amsterdam, The Netherlands, 2015; pp. 93-140.

51. Shah, D.U.; Porter, D.; Vollrath, F. Can silk become an effective reinforcing fibre? A property comparison with flax and glass reinforced composites. Compos. Sci. Technol. 2014, 101, 173-183. [CrossRef]

52. Akil, H.M.; Omar, M.F.; Mazuki, A.A.M.; Safiee, S.; Ishak, Z.A.M.; Bakar, A. Kenaf fibre reinforced composites: A review. Mater. Des. 2011, 32, 4107-4121. [CrossRef]

53. Haque, A.N.M.A.; Remadevi, R.; Naebe, M. Lemongrass (Cymbopogon): A review on its structure, properties, applications and recent developments. Cellulose 2018, 25, 5455-5477. [CrossRef]

54. Hori, K.; Flavier, M.E.; Kuga, S.; Lam, T.B.T.; Iiyama, K. Excellent oil absorbent kapok [Ceiba pentandra (L.) Gaertn.] fibre: Fibre structure, chemical characteristics, and application. J. Wood Sci. 2000, 46, 401-404. [CrossRef]

55. Huda, M.S.; Drzal, L.T.; Ray, D.; Mohanty, A.K.; Mishra, M. Natural-fibre composites in the automotive sector. In Properties and Performance of Natural-Fibre Composites; Elsevier: Amsterdam, The Netherlands, 2008; pp. 221-268.

56. Gholampour, A.; Ozbakkaloglu, T. A Review of Natural Fibre Composites: Properties, Modification and Processing Techniques, Characterization, Applications. J. Mater. Sci. 2020, 55, 829-892. [CrossRef]

57. Pothan, L.A. Natural Fibre Reinforced Polymer Composite from Macro to Nanoscale; Old City Publishing: Philadelphia, PA, USA, 2009.

58. UNCTAD United Nations Conference on Trade and Development; Trust Fund on Market Information on Agricultural Commodities. Banana An Infocom Commodity Profile UNCTAD Trust Fund on Market Information on Agricultural Commodities; United Nations (UN): New York, NY, USA; Geneva, Switzerland, 2016; pp. 1-21.

59. Reddy, N.; Yang, Y. Fibres from sugarcane bagasse. In Innovative Biofibres from Renewable Resources; Springer: Berlin/Heidelberg, Germany, 2015; pp. 29-30.

60. Todkar, S.S.; Patil, S.A. Review on mechanical properties evaluation of Pineapple Leaf Fibre (PALF) reinforced polymer composites. Compos. Part B Eng. 2019, 174, 106927. [CrossRef]

61. Balasubramanian, M. Introduction to composite materials. In Fibrous and Textile Materials for Composite Applications; Springer: Berlin/Heidelberg, Germany, 2016; pp. 1-38.

62. Yao, S.S.; Jin, F.L.; Rhee, K.Y.; Hui, D.; Park, S.J. Recent advances in carbon-fibre-reinforced thermoplastic composites: A review. Compos. Part B Eng. 2018, 142, 241-250. [CrossRef]

63. Gouzman, I.; Atar, N.; Grossman, E.; Verker, R.; Bolker, A.; Pokrass, M.; Sultan, S.; Sinwani, O.; Wagner, A.; Lück, T.; et al. 3D Printing of Bismaleimides: From New Ink Formulation to Printed Thermosetting Polymer Objects. Adv. Mater. Technol. 2019, 4, 1900368. [CrossRef]

64. Yun, I.S.; Hwang, S.W.; Shim, J.K.; Seo, K.H. A study on the thermal and mechanical properties of poly (butylene succinate)/thermoplastic starch binary blends. Int. J. Precis. Eng. Manuf. Technol. 2016, 3, $289-296$. [CrossRef]

65. Ke, C.-H.; Li, J.; Fang, K.-Y.; Zhu, Q.-L.; Zhu, J.; Yan, Q.; Wang, Y.-Z. Synergistic effect between a novel hyperbranched charring agent and ammonium polyphosphate on the flame retardant and anti-dripping properties of polylactide. Polym. Degrad. Stab. 2010, 95, 763-770. [CrossRef]

66. Stapulionienè, R.; Vaitkus, S.; Vejjelis, S.; Sankauskaite, A. Investigation of thermal conductivity of natural fibres processed by different mechanical methods. Int. J. Precis. Eng. Manuf. 2016, 17, 1371-1381. [CrossRef]

67. Marques, A. Fibrous materials reinforced composites production techniques. In Fibrous and Composite Materials for Civil Engineering Applications; Elsevier: Amsterdam, The Netherlands, 2011; pp. 191-215.

68. Rajak, D.K.; Pagar, D.D.; Menezes, P.L.; Linul, E. Fiber-Reinforced Polymer Composites: Manufacturing, Properties, and Applications. Polymers 2019, 11, 1667. [CrossRef]

69. Hollaway, L. Handbook of Polymer Composites for Engineers; Elsevier: Amsterdam, The Netherlands, 1994.

70. Crutchlow, R. Changing from open to closed moulding. Reinf. Plast. 2004, 48, 40-41. [CrossRef]

71. Leong, Y.W.; Thitithanasarn, S.; Yamada, K.; Hamada, H. 8-Compression and Injection Molding Techniques for Natural Fibre Composites; Hodzic, A., Shanks, R.T.B.N.F.C., Eds.; Woodhead Publishing: Cambridge, UK, 2014; pp. 216-232. 
72. Fabrication Methods. Available online: https://www.compositesworld.com/articles/fabrication-methods (accessed on 25 August 2020).

73. Summerscales, J.; Grove, S. Manufacturing Methods for Natural Fibre Composites; Elsevier: Amsterdam, The Netherlands, 2014; pp. 176-215.

74. Open Molding Processes Composites Lab. Available online: http://compositeslab.com/compositesmanufacturing-processes/open-molding/ (accessed on 8 September 2020).

75. Idicula, M.; Boudenne, A.; Umadevi, L.; Ibos, L.; Candau, Y.; Thomas, S. Thermophysical properties of natural fibre reinforced polyester composites. Compos. Sci. Technol. 2006, 66, 2719-2725. [CrossRef]

76. Mazumdar, S. Composites Manufacturing; CRC Press: Boca Raton, FL, USA, 2001.

77. Cripps, D.; Searle, T.; Summerscales, J. Open Mold Techniques for Thermoset Composites; Elsevier: Amsterdam, The Netherlands, 2000; pp. 737-761.

78. Brouwer, W.; Van Herpt, E.; Labordus, M. Vacuum injection moulding for large structural applications. Compos. Part A: Appl. Sci. Manuf. 2003, 34, 551-558. [CrossRef]

79. Loff, A. Choosing the right closed moulding process. Reinf. Plast. 2004, 48, 46-48. [CrossRef]

80. Cawood, M.; Smith, G. A compression moulding technique for thick sheets of thermoplastics. Polym. Test. 1980, 1, 3-7. [CrossRef]

81. Oksman, K.; Skrifvars, M.; Selin, J.-F. Natural fibres as reinforcement in polylactic acid (PLA) composites. Compos. Sci. Technol. 2003, 63, 1317-1324. [CrossRef]

82. Breukelaar, A.H. Introduction: On method. Hist. Episcop. Auth. Sixth-Century Gaul 1994, 2, $135-139$. [CrossRef]

83. Saleem, Z.; Rennebaum, H.; Pudel, F.; Grimm, E. Treating bast fibres with pectinase improves mechanical characteristics of reinforced thermoplastic composites. Compos. Sci. Technol. 2008, 68, 471-476. [CrossRef]

84. Thomason, J.L. Interfacial strength in thermoplastic composites-At last an industry friendly measurement method? Compos. Part A Appl. Sci. Manuf. 2002, 33, 1283-1288. [CrossRef]

85. Rudd, C.D.; Long, A.C.; Kendall, K.N.; Mangin, C.G.E. Liquid Moulding Technologies; Elsevier: Amsterdam, The Netherlands, 1997.

86. Gunge, A.; Koppad, P.G.; Nagamadhu, M.; Kivade, S.; Murthy, K.S. Study on mechanical properties of alkali treated plain woven banana fabric reinforced biodegradable composites. Compos. Commun. 2019, 13, 47-51. [CrossRef]

87. Elkington, M.; Bloom, D.; Ward, C.; Chatzimichali, A.; Potter, K. Hand layup: Understanding the manual process. Adv. Manuf. Polym. Compos. Sci. 2015, 1, 138-151. [CrossRef]

88. Sozer, E.; Simacek, P.; Advani, S. Resin transfer molding (RTM) in polymer matrix composites. In Manufacturing Techniques for Polymer Matrix Composites (PMCs); Elsevier: Amsterdam, The Netherlands, 2012; pp. 245-309.

89. Hsiao, K.-T.; Heider, D. Vacuum Assisted Resin Transfer Molding (VARTM) in Polymer Matrix Composites; Elsevier: Amsterdam, The Netherlands, 2012; pp. 310-347.

90. Qi, B.; Raju, J.; Kruckenberg, T.; Stanning, R. A resin film infusion process for manufacture of advanced composite structures. Compos. Struct. 1999, 47, 471-476. [CrossRef]

91. Sidewell, D.R. Hand lay-up and bag molding. In Handbook of Composites; Springer Science and Business Media LLC: New York, NY, USA, 1998; pp. 352-377.

92. Zweben, C. Composite materials. Mech. Eng. Handb. 2006, 1, 380-417. [CrossRef]

93. Awan, F.S.; Fakhar, M.A.; Khan, L.A.; Zaheer, U.; Khan, A.F.; Subhani, T. Interfacial mechanical properties of carbon nanotube-deposited carbon fiber epoxy matrix hierarchical composites. Compos. Interfaces 2018, 25, 681-699. [CrossRef]

94. Bender, D.; Schuster, J.; Heider, D. Flow rate control during vacuum-assisted resin transfer molding (VARTM) processing. Compos. Sci. Technol. 2006, 66, 2265-2271. [CrossRef]

95. Yoon, M.K.; Baidoo, J.; Gillespie, J.W.; Heider, D.J.; Hsiao, K.-T. Vacuum Assisted Resin Transfer Molding (VARTM) Process Incorporating Gravitational Effects: A Closed-form Solution. J. Compos. Mater. 2005, 39, 2227-2242. [CrossRef]

96. Kedari, V.R.; Farah, B.I.; Hsiao, K.-T. Effects of vacuum pressure, inlet pressure, and mold temperature on the void content, volume fraction of polyester/e-glass fiber composites manufactured with VARTM process. J. Compos. Mater. 2011, 45, 2727-2742. [CrossRef] 
97. Yalcinkaya, M.A.; Sozer, E.M.; Altan, M.C. Fabrication of high quality composite laminates by pressurized and heated-VARTM. Compos. Part A Appl. Sci. Manuf. 2017, 102, 336-346. [CrossRef]

98. Ferreira, F.V.; Pinheiro, I.F.; Mariano, M.; Cividanes, L.S.; Costa, J.C.; Nascimento, N.R.; Kimura, S.P.; Neto, J.C.; Lona, L.M. Environmentally friendly polymer composites based on PBAT reinforced with natural fibers from the amazon forest. Polym. Compos. 2019, 40, 3351-3360. [CrossRef]

99. Gassan, J. A study of fibre and interface parameters affecting the fatigue behaviour of natural fibre composites. Compos. Part A Appl. Sci. Manuf. 2002, 33, 369-374. [CrossRef]

100. Cha, Y.-H.; Kim, K.-S.; Kim, D.-J. Evaluation on the fracture toughness and strength of fiber reinforced brittle matrix composites. KSME Int. J. 1998, 12, 370-379. [CrossRef]

101. Shetty, M.R.; Kumar, K.R.V.; Sudhir, S.; Raghu, P.; Madhuranath, A.D.; Rao, R.M.V.G.K. Effect of Fibre Orientation on Mode-I Interlaminar Fracture Toughness of Glass Epoxy Composites. J. Reinf. Plast. Compos. 2000, 19, 606-620. [CrossRef]

102. Bilisik, K.; Karaduman, N.S.; Sapanci, E. Flexural characterization of 3D prepreg/stitched carbon/epoxy/ multiwalled carbon nanotube preforms and composites. J. Compos. Mater. 2018, 53, 563-577. [CrossRef]

103. Bilisik, K.; Karaduman, N.S.; Sapanci, E. Tensile properties of nanoprepreg/nanostitched 3D carbon/epoxy MWCNTs composites. Mech. Mater. 2019, 128, 11-23. [CrossRef]

104. Bilisik, K.; Karaduman, N.; Sapanci, E. Short-beam shear of nanoprepreg/nanostitched three-dimensional carbon/epoxy multiwall carbon nanotube composites. J. Compos. Mater. 2019, 54, 311-329. [CrossRef]

105. Tan, C.; Peng, J.; Lin, W.; Xing, Y.; Xu, K.; Wu, J.; Chen, M. Role of surface modification and mechanical orientation on property enhancement of cellulose nanocrystals/polymer nanocomposites. Eur. Polym. J. 2015, 62, 186-197. [CrossRef]

106. Habibi, Y.; Lucia, L.A.; Rojas, O.J. Cellulose Nanocrystals: Chemistry, Self-Assembly, and Applications. Chem. Rev. 2010, 110, 3479-3500. [CrossRef]

107. Henrique, M.A.; Neto, W.P.F.; Silvério, H.A.; Martins, D.F.; Gurgel, L.V.A.; Barud, H.D.S.; De Morais, L.C.; Pasquini, D. Kinetic study of the thermal decomposition of cellulose nanocrystals with different polymorphs, cellulose I and II, extracted from different sources and using different types of acids. Ind. Crop. Prod. 2015, 76, 128-140. [CrossRef]

108. Azwa, Z.N.; Yousif, B.F.; Manalo, A.C.; Karunasena, W. A review on the degradability of polymeric composites based on natural fibres. Mater. Des. 2013, 47, 424-442. [CrossRef]

109. Juntaro, J.; Pommet, M.; Mantalaris, A.; Shaffer, M.; Bismarck, A. Nanocellulose enhanced interfaces in truly green unidirectional fibre reinforced composites. Compos. Interfaces 2007, 14, 753-762. [CrossRef]

110. Kabir, M.M.; Wang, H.; Aravinthan, T.; Cardona, F.; Lau, K.-T. Effects of natural fibre surface on composite properties: A review. In Proceedings of the 1st International Postgraduate Conference on Engineering, Designing and Developing the Built Environment for Sustainable Wellbeing, Brisbane, Australia, 27-29 April 2011; pp. 94-99.

111. El-Sabbagh, A. Effect of coupling agent on natural fibre in natural fibre/polypropylene composites on mechanical and thermal behaviour. Compos. Part B Eng. 2014, 57, 126-135. [CrossRef]

112. Kabir, M.M.; Wang, H.; Lau, K.T.; Cardona, F. Chemical treatments on plant-based natural fibre reinforced polymer composites: An overview. Compos. Part B Eng. 2012, 43, 2883-2892. [CrossRef]

113. Rokbi, M.; Osmani, H.; Imad, A.; Benseddiq, N. Effect of Chemical treatment on Flexure Properties of Natural Fiber-reinforced Polyester Composite. Procedia Eng. 2011, 10, 2092-2097. [CrossRef]

114. Yan, L.; Chouw, N.; Yuan, X. Improving the mechanical properties of natural fibre fabric reinforced epoxy composites by alkali treatment. J. Reinf. Plast. Compos. 2012, 31, 425-437. [CrossRef]

115. Boopathi, L.; Sampath, P.; Mylsamy, K. Investigation of physical, chemical and mechanical properties of raw and alkali treated Borassus fruit fiber. Compos. Part B Eng. 2012, 43, 3044-3052. [CrossRef]

116. Cai, M.; Takagi, H.; Nakagaito, A.N.; Li, Y.; Waterhouse, G.I. Effect of alkali treatment on interfacial bonding in abaca fiber-reinforced composites. Compos. Part A Appl. Sci. Manuf. 2016, 90, 589-597. [CrossRef]

117. Reddy, K.O.; Maheswari, C.U.; Shukla, M.; Jung-Il, S.; Rajulu, A.V. Tensile and structural characterization of alkali treated Borassus fruit fine fibers. Compos. Part B Eng. 2013, 44, 433-438. [CrossRef]

118. Thamae, T.; Baillie, C. Influence of fibre extraction method, alkali and silane treatment on the interface of Agave americana waste HDPE composites as possible roof ceilings in Lesotho. Compos. Interfaces 2007, 14, 821-836. [CrossRef] 
119. Asumani, O.; Reid, R.; Paskaramoorthy, R. The effects of alkali-silane treatment on the tensile and flexural properties of short fibre non-woven kenaf reinforced polypropylene composites. Compos. Part A Appl. Sci. Manuf. 2012, 43, 1431-1440. [CrossRef]

120. Goriparthi, B.K.; Suman, K.; Rao, N.M. Effect of fiber surface treatments on mechanical and abrasive wear performance of polylactide/jute composites. Compos. Part A Appl. Sci. Manuf. 2012, 43, 1800-1808. [CrossRef]

121. Herrera-Franco, P.J.; Valadez-Gonzalez, A. Mechanical properties of continuous natural fibre-reinforced polymer composites. Compos. Part A Appl. Sci. Manuf. 2004, 35, 339-345. [CrossRef]

122. Bisanda, E.; Ansell, M. The effect of silane treatment on the mechanical and physical properties of sisal-epoxy composites. Compos. Sci. Technol. 1991, 41, 165-178. [CrossRef]

123. Liu, Y.; Lv, X.; Bao, J.; Xie, J.; Tang, X.; Che, J.; Ma, Y.H.; Tong, J. Characterization of silane treated and untreated natural cellulosic fibre from corn stalk waste as potential reinforcement in polymer composites. Carbohydr. Polym. 2019, 218, 179-187. [CrossRef] [PubMed]

124. Tserki, V.; Zafeiropoulos, N.; Simon, F.; Panayiotou, C. A study of the effect of acetylation and propionylation surface treatments on natural fibres. Compos. Part A Appl. Sci. Manuf. 2005, 36, 1110-1118. [CrossRef]

125. Naik, J.; Mishra, S. Esterification Effect of Maleic Anhydride on Surface and Volume Resistivity of Natural Fiber/Polystyrene Composites. Polym. Technol. Eng. 2007, 46, 537-540. [CrossRef]

126. Pasquini, D.; Teixeira, E.D.M.; Curvelo, A.A.D.S.; Belgacem, M.N.; Dufresne, A. Surface esterification of cellulose fibres: Processing and characterisation of low-density polyethylene/cellulose fibres composites. Compos. Sci. Technol. 2008, 68, 193-201. [CrossRef]

127. Dantas, L.G.; Motta, L.A.D.C.; Pasquini, D.; Vieira, J.G. Surface Esterification of Sisal Fibres for use as Reinforcement in Cementitious Matrix. Mater. Res. 2019, 22. [CrossRef]

128. Wirawan, R.; Zainudin, E.S.; Sapuan, S.M. Mechanical properties of natural fibre reinforced PVC composites: A review. Sains Malays. 2009, 38, 531-535.

129. Ramnath, B.V.; Kokan, S.J.; Raja, R.N.; Sathyanarayanan, R.; Elanchezhian, C.; Prasad, A.R.; Manickavasagam, V. Evaluation of mechanical properties of abaca-jute-glass fibre reinforced epoxy composite. Mater. Des. 2013, 51, 357-366. [CrossRef]

130. Abu Bakar, M.A.; Ahmad, S.; Kuntjoro, W.; Kasolang, S. Effect of carbon fibre ratio to the impact properties of hybrid kenaf/carbon fibre reinforced epoxy composites. Appl. Mech. Mater. 2013, 393, 136-139. [CrossRef]

131. Aslan, M.; Tufan, M.; Küçükömeroğlu, T. Tribological and mechanical performance of sisal-filled waste carbon and glass fibre hybrid composites. Compos. Part B Eng. 2018, 140, 241-249. [CrossRef]

132. Khanam, P.N.; Khalil, H.P.S.A.; Jawaid, M.; Reddy, G.R.; Narayana, C.S.; Naidu, S.V. Sisal/carbon fibre reinforced hybrid composites: Tensile, flexural and chemical resistance properties. J. Polym. Environ. 2010, 18, 727-733. [CrossRef]

133. Goud, G.; Rao, R.N. Mechanical and electrical performance of roystonea regia/glass fibre reinforced epoxy hybrid composites. Bull. Mater. Sci. 2012, 35, 595-599. [CrossRef]

134. Bharat, K.R.; Abhishek, S.; Palanikumar, K. Mechanical property analysis on sandwich structured hybrid composite made from natural fibre, glass fibre and ceramic fibre wool reinforced with epoxy resin. IOP Conf. Ser. Mater. Sci. Eng. 2017, 205, 12015. [CrossRef]

135. Atiqah, A.; Jawaid, M.; Jawaid, M.; Ishak, M.; Ansari, M.; Ilyas, R. Physical and thermal properties of treated sugar palm/glass fibre reinforced thermoplastic polyurethane hybrid composites. J. Mater. Res. Technol. 2019, 8, 3726-3732. [CrossRef]

136. Misri, S.; Leman, Z.; Sapuan, S.M.; Ishak, M.R. Mechanical properties and fabrication of small boat using woven glass/sugar palm fibres reinforced unsaturated polyester hybrid composite. IOP Conf. Ser. Mater. Sci. Eng. 2010, 11, 012015. [CrossRef]

137. Wambua, P.; Ivens, J.; Verpoest, I. Natural fibres: Can they replace glass in fibre reinforced plastics? Compos. Sci. Technol. 2003, 63, 1259-1264. [CrossRef]

138. Cavalcanti, D.; Banea, M.D.; Neto, J.; Lima, R.; Da Silva, L.; Carbas, R. Mechanical characterization of intralaminar natural fibre-reinforced hybrid composites. Compos. Part B Eng. 2019, 175, 107149. [CrossRef]

139. Singh, J.I.P.; Singh, S.; Dhawan, V. Effect of curing temperature on mechanical properties of natural fiber reinforced polymer composites. J. Nat. Fibers 2017, 15, 687-696. [CrossRef]

140. Baghaei, B.; Skrifvars, M.; Salehi, M.; Bashir, T.; Rissanen, M.; Nousiainen, P. Novel aligned hemp fibre reinforcement for structural biocomposites: Porosity, water absorption, mechanical performances and viscoelastic behaviour. Compos. Part A Appl. Sci. Manuf. 2014, 61,1-12. [CrossRef] 
141. Dhakal, H.; Zhang, Z.; Guthrie, R.; Mac Mullen, J.; Bennett, N. Development of flax/carbon fibre hybrid composites for enhanced properties. Carbohydr. Polym. 2013, 96,1-8. [CrossRef]

142. Torres, F.G.; Cubillas, M. Study of the interfacial properties of natural fibre reinforced polyethylene. Polym. Test. 2005, 24, 694-698. [CrossRef]

143. Rao, K.M.M.; Prasad, A.R. Fabrication and testing of natural fibre composites: Vakka, sisal, bamboo and banana. Mater. Des. 2010, 31, 508-513. [CrossRef]

144. Prasad, A.R.; Rao, K.M. Mechanical properties of natural fibre reinforced polyester composites: Jowar, sisal and bamboo. Mater. Des. 2011, 32, 4658-4663. [CrossRef]

145. Arrakhiz, F.; Elachaby, M.; Bouhfid, R.; Vaudreuil, S.; Essassi, M.; Qaiss, A.E.K. Mechanical and thermal properties of polypropylene reinforced with Alfa fiber under different chemical treatment. Mater. Des. 2012, 35, 318-322. [CrossRef]

146. Sreenivasan, V.; Ravindran, D.; Manikandan, V.; Narayanasamy, R. Influence of fibre treatments on mechanical properties of short Sansevieria cylindrica/polyester composites. Mater. Des. 2012, 37, 111-121. [CrossRef]

147. Arrakhiz, F.; El Achaby, M.; Kakou, A.; Vaudreuil, S.; Benmoussa, K.; Bouhfid, R.; Fassi-Fehri, O.; Qaiss, A.E.K. Mechanical properties of high density polyethylene reinforced with chemically modified coir fibers: Impact of chemical treatments. Mater. Des. 2012, 37, 379-383. [CrossRef]

148. Gironès, J.; López, J.; Mutjé, P.; Carvalho, A.J.F.; Curvelo, A.; Vilaseca, F. Natural fiber-reinforced thermoplastic starch composites obtained by melt processing. Compos. Sci. Technol. 2012, 72, 858-863. [CrossRef]

149. Wu, Y.; Xia, C.; Cai, L.; Garcia, A.C.; Shi, S.Q. Development of natural fiber-reinforced composite with comparable mechanical properties and reduced energy consumption and environmental impacts for replacing automotive glass-fiber sheet molding compound. J. Clean. Prod. 2018, 184, 92-100. [CrossRef]

150. Jawaid, M.; Khalil, H.A.; Hassan, A.; Dungani, R.; Hadiyane, A. Effect of jute fibre loading on tensile and dynamic mechanical properties of oil palm epoxy composites. Compos. Part B Eng. 2013, 45, 619-624. [CrossRef]

151. Boopalan, M.; Niranjanaa, M.; Umapathy, M.J. Study on the mechanical properties and thermal properties of jute and banana fiber reinforced epoxy hybrid composites. Compos. Part B Eng. 2013, 51, 54-57. [CrossRef]

152. Sreekala, M.S.; Kumaran, M.G.; Joseph, S.; Jacob, M.; Thomas, S. Oil palm fibre reinforced phenol formaldehyde composites: Influence of fibre surface modifications on the mechanical performance. Appl. Compos. Mater. 2000, 7, 295-329. [CrossRef]

153. Yan, L.; Chouw, N.; Huang, L.; Kasal, B. Effect of alkali treatment on microstructure and mechanical properties of coir fibres, coir fibre reinforced-polymer composites and reinforced-cementitious composites. Constr. Build. Mater. 2016, 112, 168-182. [CrossRef]

154. Kandola, B.K.; Mistik, S.; Pornwannachai, W.; Anand, S. Natural fibre-reinforced thermoplastic composites from woven-nonwoven textile preforms: Mechanical and fire performance study. Compos. Part B Eng. 2018, 153, 456-464. [CrossRef]

155. Singh, J.I.P.; Dhawan, V.; Singh, S.; Jangid, K. Study of effect of surface treatment on mechanical properties of natural fiber reinforced composites. Mater. Today Proc. 2017, 4, 2793-2799. [CrossRef]

156. Abdulkareem, S.A.; Adeniyi, A.G. Tensile and water absorbing properties of natural fibre reinforced plastic composites from waste polystyrene and rice husk. ABUAD J. Eng. Res. Dev. 2018, 1, 199-204.

157. Chandramohan, D.; Murali, B.; Vasantha-Srinivasan, P.; Kumar, S.D. Mechanical, moisture absorption, and abrasion resistance properties of bamboo-jute-glass fiber composites. J. Bio-Tribo-Corrosion 2019, 5, 66. [CrossRef]

158. Reddy, K.H.; Reddy, R.M.; Ramesh, M.; Krishnudu, D.M.; Reddy, B.M.; Rao, H.R. Impact of alkali treatment on characterization of Tapsi (Sterculia Urens) natural bark fiber reinforced polymer composites. J. Nat. Fibers 2019, 1-12. [CrossRef]

159. Gunti, R.; Prasad, A.R.; Gupta, A. Mechanical and degradation properties of natural fiber-reinforced PLA composites: Jute, sisal, and elephant grass. Polym. Compos. 2016, 39, 1125-1136. [CrossRef]

160. Gassan, J. Effects of corona discharge and UV treatment on the properties of jute-fibre epoxy composites. Compos. Sci. Technol. 2000, 60, 2857-2863. [CrossRef]

161. Marais, S.; Gouanvé, F.; Bonnesoeur, A.; Grenet, J.; Poncin-Epaillard, F.; Morvan, C.; Métayer, M. Unsaturated polyester composites reinforced with flax fibers: Effect of cold plasma and autoclave treatments on mechanical and permeation properties. Compos. Part A Appl. Sci. Manuf. 2005, 36, 975-986. [CrossRef] 
162. Beg, M.D.H.; Pickering, K. Mechanical performance of kraft fibre reinforced polypropylene composites: Influence of fibre length, fibre beating and hygrothermal ageing. Compos. Part A Appl. Sci. Manuf. 2008, 39, 1748-1755. [CrossRef]

163. Ray, D.; Sarkar, B.; Rana, A.; Bose, N. The mechanical properties of vinylester resin matrix composites reinforced with alkali-treated jute fibres. Compos. Part A Appl. Sci. Manuf. 2001, 32, 119-127. [CrossRef]

164. Suardana, N.P.G.; Piao, Y.; Lim, J.K. Mechanical properties of HEMP fibres and HEMP/PP composites: Effects of chemical surface treatment. Mater. Phys. Mech. 2011, 11, 1-8.

165. Bledzki, A.K.; Mamun, A.A.; Lucka-Gabor, M.; Gutowski, V.S. The effects of acetylation on properties of flax fibre and its polypropylene composites. Express Polym. Lett. 2008, 2, 413-422. [CrossRef]

166. Manikandan Nair, K.C.; Diwan, S.M.; Thomas, S. Tensile properties of short sisal fibre reinforced polystyrene composites. J. Appl. Polym. Sci. 1996, 60, 1483-1497. [CrossRef]

167. Hong, C.K.; Kim, N.; Kang, S.L.; Nah, C.; Lee, Y.-S.; Cho, B.-H.; Ahn, J.-H. Mechanical properties of maleic anhydride treated jute fibre/polypropylene composites. Plast. Rubber Compos. 2008, 37, 325-330. [CrossRef]

168. Pickering, K.L.; Li, Y.; Farrell, R.L.; Lay, M. Interfacial modification of hemp fibre reinforced composites using fungal and alkali treatment. J. Biobased Mater. Bioenergy 2007, 1, 109-117. [CrossRef]

169. Agarwal, B.D.; Broutman, L.J.; Chandrashekhara, K. Analysis and Performance of Fibre Composites; John Wiley \& Sons: Hoboken, NJ, USA, 2006.

170. Nair, A.B.; Joseph, R. Eco-friendly bio-composites using natural rubber (NR) matrices and natural fibre reinforcements. In Chemistry, Manufacture and Applications of Natural Rubber; Elsevier: Amsterdam, The Netherlands, 2014; pp. 249-283.

171. Yang, Z.; Peng, H.; Wang, W.; Liu, T. Crystallization behavior of poly( $\varepsilon$-caprolactone)/layered double hydroxide nanocomposites. J. Appl. Polym. Sci. 2010, 116, 2658-2667. [CrossRef]

172. Ozbakkaloglu, T.; Vincent, T. Axial compressive behavior of circular high-strength concrete-filled FRP tubes. J. Compos. Constr. 2014, 18, 04013037. [CrossRef]

173. Ozbakkaloglu, T. Compressive behavior of concrete-filled FRP tube columns: Assessment of critical column parameters. Eng. Struct. 2013, 51, 188-199. [CrossRef]

174. Zhao, Y.Q.; Zhou, Y.; Huang, Z.-M.; Batra, R. Experimental and micromechanical investigation of T300/7901 unidirectional composite strength. Polym. Compos. 2018, 40, 2639-2652. [CrossRef]

175. Abdellaoui, H.; Raji, M.; Essabir, H.; Bouhfid, R.; el kacem Qaiss, A. 6-Mechanical behavior of carbon/natural fibre-based hybrid composites. In Woodhead Publishing Series in Composites Science and Engineering; Jawaid, M., Thariq, M., Saba Mechanical and Physical Testing of Biocomposites, Fibre-Reinforced Composites and Hybrid Composites, Eds.; Woodhead Publishing: Cambridge, UK, 2019; pp. 103-122.

176. Ghalia, M.A.; Abdelrasoul, A. 7-Compressive and fracture toughness of natural and synthetic fibre-reinforced polymer. In Woodhead Publishing Series in Composites Science and Engineering; Jawaid, M., Thariq, M., Saba Mechanical and Physical Testing of Biocomposites, Fibre-Reinforced Composites and Hybrid Composites, Eds.; Woodhead Publishing: Cambridge, UK, 2019; pp. 123-140.

177. Rajak, D.K.; Pagar, D.D.; Kumar, R.; Pruncu, C.I. Recent progress of reinforcement materials: A comprehensive overview of composite materials. J. Mater. Res. Technol. 2019, 8, 6354-6374. [CrossRef]

178. Battegazzore, D.; Abt, T.; Maspoch, M.L.; Frache, A. Multilayer cotton fabric bio-composites based on PLA and PHB copolymer for industrial load carrying applications. Compos. Part B Eng. 2019, 163, 761-768. [CrossRef]

179. Ku, H.; Wang, H.; Pattarachaiyakoop, N.; Trada, M. A review on the tensile properties of natural fiber reinforced polymer composites. Compos. Part B Eng. 2011, 42, 856-873. [CrossRef]

180. Goud, G.; Rao, R.N. Effect of fibre content and alkali treatment on mechanical properties of Roystonea regia-reinforced epoxy partially biodegradable composites. Bull. Mater. Sci. 2011, 34, 1575-1581. [CrossRef]

181. Mokhothu, T.H.; John, M.J. Review on hygroscopic aging of cellulose fibres and their biocomposites. Carbohydr. Polym. 2015, 131, 337-354. [CrossRef]

182. Sreekala, M.; Thomas, S. Effect of fibre surface modification on water-sorption characteristics of oil palm fibres. Compos. Sci. Technol. 2003, 63, 861-869. [CrossRef]

183. Bismarck, A.; Aranberri-Askargorta, I.; Lampke, T.; Wielage, B.; Stamboulis, A.; Shenderovich, I.; Limbach, H.-H. Surface characterization of flax, hemp and cellulose fibers; Surface properties and the water uptake behavior. Polym. Compos. 2002, 23, 872-894. [CrossRef] 
184. Matthews, F.L.; Rawlings, R.D. Composite Materials: Engineering and Science; CRC Press: Boca Raton, FL, USA, 1999.

185. Sumaila, M.; Amber, I.; Bawa, M. Effect of fibre length on the physical and mechanical properties of random oreinted, nonwoven short banana (musa balbisiana) fibre/epoxy composite. Asian J. Nat. Appl. Sci. 2013, 2, 39-49.

186. Young, R. Composite Micromechanics; Elsevier: Amsterdam, The Netherlands, 2015; pp. 3-23.

187. Ferreira, F.V.; Menezes, B.R.C.; Franceschi, W.; Lozano, K.; Cividanes, L.S.; Coutinho, A.R.; Thim, G.P. Influence of carbon nanotube concentration and sonication temperature on mechanical properties of HDPE/CNT nanocomposites. Full-Nanotub. Carbon Nanostruct. 2017, 25, 531-539. [CrossRef]

188. Ho, M.P.; Wang, H.; Lee, J.H.; Ho, C.K.; Lau, K.T.; Leng, J.; Hui, D. Critical factors on manufacturing processes of natural fibre composites. Compos. Part B Eng. 2012, 43, 3549-3562. [CrossRef]

189. Sinha, E.; Panigrahi, S. Effect of plasma treatment on structure, wettability of jute fiber and flexural strength of its composite. J. Compos. Mater. 2009, 43, 1791-1802. [CrossRef]

190. Mohanty, A.K.; Misra, M.; Drzal, L.T. Surface modifications of natural fibers and performance of the resulting biocomposites: An overview. Compos. Interfaces 2001, 8, 313-343. [CrossRef]

191. Mukhopadhyay, S.; Fangueiro, R. Physical modification of natural fibers and thermoplastic films for composites-A review. J. Thermoplast. Compos. Mater. 2009, 22, 135-162. [CrossRef]

192. Thakur, V.K.; Singha, A.S. (Eds.) Surface Modification of Biopolymers; John Wiley \& Sons, Inc.: Hoboken, NJ, USA, 2015. [CrossRef]

193. Gulati, D.; Sain, M. Fungal-modification of natural fibers: A novel method of treating natural fibers for composite reinforcement. J. Polym. Environ. 2006, 14, 347-352. [CrossRef]

194. Li, X.; Tabil, L.G.; Panigrahi, S. Chemical treatments of natural fiber for use in natural fiber-reinforced composites: A review. J. Polym. Environ. 2007, 15, 25-33. [CrossRef]

195. Edalatmanesh, M.; Sain, M.; Liss, S.N.; Kucharczyk, P.; Sedlarik, V.; Miskolczi, N.; Szakacs, H.; Kitano, T. Enzymatic modification of secondary sludge by lipase and laccase to improve the nylon/sludge composite properties. J. Reinf. Plast. Compos. 2012, 31, 179-188. [CrossRef]

196. Medina, L.; Schledjewski, R.; Schlarb, A.K. Process related mechanical properties of press molded natural fiber reinforced polymers. Compos. Sci. Technol. 2009, 69, 1404-1411. [CrossRef]

197. Epstein, W.M. Book Review: Quixote's Ghost: The Right, the Liberati, and the Future of Social Policy. Res. Soc. Work. Pract. 2006, 16, 238-240. [CrossRef]

198. Rao, S.; Bhattacharyya, D.; Jayaraman, K.; Fernyhough, A. Influence of material parameters on the mechanical properties of extruded sisal fibre-polypropylene composites. Int. SAMPE Symp. Exhib. 2008, 52, 1625-1640.

199. Shih, Y.F.; Huang, C.C. Polylactic acid (PLA)/banana fibre (BF) biodegradable green composites. J. Polym. Res. 2011, 18, 2335-2340. [CrossRef]

200. Fazita, M.N.; Jayaraman, K.; Bhattacharyya, D.; Haafiz, M.M.; Saurabh, C.K.; Hussin, M.H.; Khalil, H.A. Green composites made of bamboo fabric and poly (lactic) acid for packaging applications-A review. Materials 2016, 9, 435. [CrossRef] [PubMed]

201. Guo, W.; Tao, J.; Yang, C.; Song, C.; Geng, W.; Li, Q.; Wang, Y.; Kong, M.; Wang, S. Introduction of environmentally degradable parameters to evaluate the biodegradability of biodegradable polymers. PLOS ONE 2012, 7, e38341. [CrossRef] [PubMed]

202. Ren, P.; Shen, T.; Wang, F.; Wang, X.; Zhang, Z. Study on biodegradable starch/OMMT nanocomposites for packaging applications. J. Polym. Environ. 2009, 17, 203-207. [CrossRef]

203. Tokiwa, Y.; Calabia, B.P. Biodegradability and biodegradation of poly(lactide). Appl. Microbiol. Biotechnol. 2006, 72, 244-251. [CrossRef] [PubMed]

204. Muniyasamy, S.; Reddy, M.M.; Misra, M.; Mohanty, A.K. Biodegradable green composites from bioethanol co-product and poly(butylene adipate-co-terephthalate). Ind. Crop. Prod. 2013, 43, 812-819. [CrossRef]

205. Way, C.; Dean, K.; Wu, D.Y.; Palombo, E.A. Biodegradation of sequentially surface treated lignocellulose reinforced polylactic acid composites: Carbon dioxide evolution and morphology. Polym. Degrad. Stab. 2012, 97, 430-438. [CrossRef]

206. Chattopadhyay, S.K.; Singh, S.; Pramanik, N.; Niyogi, U.K.; Khandal, R.K.; Uppaluri, R.; Ghosal, A.K. Biodegradability studies on natural fibres reinforced polypropylene composites. J. Appl. Polym. Sci. 2011, 121, 2226-2232. [CrossRef] 
207. Shibata, M.; Oyamada, S.; Kobayashi, S.-I.; Yaginuma, D. Mechanical properties and biodegradability of green composites based on biodegradable polyesters and lyocell fabric. J. Appl. Polym. Sci. 2004, 92, 3857-3863. [CrossRef]

208. Ochi, S. Mechanical properties of kenaf fibres and kenaf/PLA composites. Mech. Mater. 2008, 40, 446-452. [CrossRef]

209. Wu, C.-S.; Liao, H.-T.; Cai, Y.-X. Characterisation, biodegradability and application of palm fibre-reinforced polyhydroxyalkanoate composites. Polym. Degrad. Stab. 2017, 140, 55-63. [CrossRef]

210. Mittal, M.; Chaudhary, R. Biodegradability and mechanical properties of pineapple leaf/coir Fibre reinforced hybrid epoxy composites. Mater. Res. Express 2019, 6, 045301. [CrossRef]

211. Huang, Z.; Qian, L.; Yin, Q.; Yu, N.; Liu, T.; Tian, D. Biodegradability studies of poly (butylene succinate) composites filled with sugarcane rind fibre. Polym. Test. 2018, 68, 319-326. [CrossRef]

212. Fernandes, T.F. Preparation and characterization of novel biodegradable composites based on acylated cellulose fibres and poly (ethylene sebacate). Compos. Sci. Technol. 2011, 71, 1908-1913. [CrossRef]

213. Yussuf, A.A.; Massoumi, I.; Hassan, A. Comparison of polylactic acid/kenaf and polylactic acid/rise husk composites: The influence of the natural fibers on the mechanical, thermal and biodegradability properties. J. Polym. Environ. 2010, 18, 422-429. [CrossRef]

214. Pradhan, R.; Misra, M.; Erickson, L.; Mohanty, A. Compostability and biodegradation study of PLA-wheat straw and PLA-soy straw based green composites in simulated composting bioreactor. Bioresour. Technol. 2010, 101, 8489-8491. [CrossRef]

215. Zuo, J.; Chung, T.-S.; O’Brien, G.S.; Kosar, W.; Chung, T.-S. Hydrophobic/hydrophilic PVDF/Ultem ${ }^{\circledR}$ dual-layer hollow fiber membranes with enhanced mechanical properties for vacuum membrane distillation. J. Membr. Sci. 2017, 523, 103-110. [CrossRef]

216. Staiger, M.P.; Tucker, N. 8-Natural-fibre composites in structural applications. In Woodhead Publishing Series in Composites Science and Engineering; Pickering, N.-F.C., Ed.; Woodhead Publishing: Cambridge, UK, 2008; pp. 269-300.

217. Ferreira, F.V.; Cividanes, L.S.; Gouveia, R.F.; Lona, L.M. An overview on properties and applications of poly(butylene adipate-co-terephthalate)-PBAT based composites. Polym. Eng. Sci. 2017, 59, E7-E15. [CrossRef]

218. Campilho, R.D.S.G. Natural Fibre Composites; CRC Press: Boca Raton, FL, USA, 2015.

219. Kozłowski, R.M. Handbook of Natural Fibres; Elsevier: Amsterdam, The Netherlands, 2012; Volume 1.

220. Venkatachalam, N.; Navaneethakrishnan, P.; Rajsekar, R.; Shankar, S. Effect of pretreatment methods on properties of natural fiber composites: A review. Polym. Polym. Compos. 2016, 24, 555-566. [CrossRef]

221. Belgacem, M.N.; Bataille, P.; Sapieha, S. Effect of corona modification on the mechanical properties of polypropylene/cellulose composites. J. Appl. Polym. Sci. 1994, 53, 379-385. [CrossRef]

222. Dong, S.; Sapieha, S.; Schreiber, H.P. Mechanical properties of corona-modified cellulose/polyethylene composites. Polym. Eng. Sci. 1993, 33, 343-346. [CrossRef]

223. Hassani, F.O.; Merbahi, N.; Oushabi, A.; Elfadili, M.; Kammouni, A.; Oueldna, N. Effects of corona discharge treatment on surface and mechanical properties of Aloe Vera fibers. Mater. Today: Proc. 2020, 24, 46-51. [CrossRef]

224. Seki, Y.; Sarikanat, M.; Sever, K.; Erden, S.; Gulec, H.A. Effect of the low and radio frequency oxygen plasma treatment of jute fiber on mechanical properties of jute fiber/polyester composite. Fibers Polym. 2010, 11, 1159-1164. [CrossRef]

225. Höcker, H. Plasma treatment of textile fibers. Pure Appl. Chem. 2002, 74, 423-427. [CrossRef]

226. Cordeiro, C.R. Plasma treatment of natural fibres to improve fibre-matrix compatibility rafael. Thesis COPPE/UFRJ 2016, 129. [CrossRef]

227. Nguyen, V.D.; Hao, J.; Wang, W. Ultraviolet weathering performance of high-density polyethylene/wood-flour composites with a basalt-fiber-included shell. Polymers 2018, 10, 831. [CrossRef]

228. Lopez, J.L.; Sain, M.; Cooper, P. Performance of natural-fiber-plastic composites under stress for outdoor applications: Effect of moisture, temperature, and ultraviolet light exposure. J. Appl. Polym. Sci. 2005, 99, 2570-2577. [CrossRef]

229. Kafi, A.-A.; Abedin, M.Z.; Beg, M.D.H.; Pickering, K.L.; Khan, M.A. Study on the mechanical properties of jute/glass fiber-reinforced unsaturated polyester hybrid composites: Effect of surface modification by ultraviolet radiation. J. Reinf. Plast. Compos. 2005, 25, 575-588. [CrossRef] 
230. Mayandi, K.; Rajini, N.; Manojprabhakar, M.; Siengchin, S.; Ayrilmis, N. 1-Recent studies on durability of natural/synthetic fibre reinforced hybrid polymer composites. In Woodhead Publishing Series in Composites Science and Engineering; Jawaid, M., Thariq, M., Saba Mechanical and Physical Testing of Biocomposites, Fibre-Reinforced Composites and Hybrid Composites, Eds.; Woodhead Publishing: Cambridge, UK, 2019; pp. 1-13.

231. Ilyas, R.A.; Sapuan, S.M.; Kadier, A.; Krishnan, S.; Atikah, M.S.N.; Ibrahim, R.; Nazrin, A.; Syafiq, R.; Misri, S.; Huzaifah, M.R.M.; et al. Mechanical Testing of Sugar Palm Fibre Reinforced Sugar Palm Biopolymer Composites. In Advanced Processing, Properties, and Applications of Starch and other Bio-based Polymers; Elsevier: Amsterdam, The Netherlands, 2020; pp. 89-110.

232. Dittenber, D.B.; GangaRao, H.V.S. Critical review of recent publications on use of natural composites in infrastructure. Compos. Part A Appl. Sci. Manuf. 2012, 43, 1419-1429. [CrossRef]

233. Padovani, J.; Legland, D.; Pernes, M.; Gallos, A.; Thomachot-Schneider, C.; Shah, D.U.; Bourmaud, A.; Beaugrand, J. Beating of hemp bast fibres: An examination of a hydro-mechanical treatment on chemical, structural, and nanomechanical property evolutions. Cellulose 2019, 26, 5665-5683. [CrossRef]

234. Song, W.; Wei, W.; Ren, C.; Zhang, S. Effect of heat treatment or alkali treatment of veneers on the mechanical properties of eucalyptus veneer/polyethylene film plywood composites. BioResources 2017, 12, 8683-8703. [CrossRef]

235. Koohestani, B.; Darban, A.K.; Mokhtari, P.; Yilmaz, E.; Darezereshki, E. Comparison of different natural fiber treatments: A literature review. Int. J. Environ. Sci. Technol. 2018, 16, 629-642. [CrossRef]

236. Diani, J.; Gall, K. Finite strain 3D thermoviscoelastic constitutive model. Society 2006. [CrossRef]

237. Shekar, H.S.; Ramachandra, M. Green Composites: A Review. Mater. Today Proc. 2018, 5, $2518-2526$. [CrossRef]

238. Cao, Y.; Sakamoto, S.; Goda, K. Effects of heat and alkali treatments on mechanical properties of kenaf fibres. In Proceedings of the International Conference on Composite Materials (ICCM), Kyoto, Japan, 8-13 July 2007.

239. Jiang, A.; Xi, J.; Wu, H. Effect of surface treatment on the morphology of sisal fibers in sisal/polylactic acid composites. J. Reinf. Plast. Compos. 2012, 31, 621-630. [CrossRef]

240. Faruk, O.; Bledzki, A.K.; Fink, H.-P.; Sain, M. Biocomposites reinforced with natural fibers: 2000-2010. Prog. Polym. Sci. 2012, 37, 1552-1596. [CrossRef]

241. Hashim, M.Y.; Roslan, M.N.; Amin, A.M.; Mujahid, A.; Zaidi, A. Mercerization treatment parameter effect on natural fibre reinforced polymer matrix composite: A brief review. World Acad. Sci. Eng. Technol. 2012, 6, $1638-1644$.

242. Kim, J.T.; Netravali, A.N. Mercerization of sisal fibers: Effect of tension on mechanical properties of sisal fiber and fiber-reinforced composites. Compos. Part A Appl. Sci. Manuf. 2010, 41, 1245-1252. [CrossRef]

243. Qin, C.; Soykeabkaew, N.; Xiuyuan, N.; Peijs, T. The effect of fibre volume fraction and mercerization on the properties of all-cellulose composites. Carbohydr. Polym. 2008, 71, 458-467. [CrossRef]

244. Goda, K.; Sreekala, M.; Gomes, A.; Kaji, T.; Ohgi, J. Improvement of plant based natural fibers for toughening green composites-Effect of load application during mercerization of ramie fibers. Compos. Part A Appl. Sci. Manuf. 2006, 37, 2213-2220. [CrossRef]

245. Kaith, B.S.; Singha, A.S.; Kumar, S.; Kalia, S. Mercerization of flax fiber improves the mechanical properties of fiber-reinforced composites. Int. J. Polym. Mater. 2008, 57, 54-72. [CrossRef]

246. Khalil, H.; Ismail, H.; Rozman, H.; Ahmad, M. The effect of acetylation on interfacial shear strength between plant fibres and various matrices. Eur. Polym. J. 2001, 37, 1037-1045. [CrossRef]

247. Tserki, V.; Panayiotou, C.; Zafeiropoulos, N.E. A Study of the effect of acetylation and propionylation on the interface of natural fibre biodegradable composites. Adv. Compos. Lett. 2005, 14, 65-71. [CrossRef]

248. Wang, H.; Kabir, M.; Lau, K.-T. Hemp Reinforced composites with alkalization and acetylation fibre treatments. Polym. Polym. Compos. 2014, 22, 247-252. [CrossRef]

249. Khalil, H.A.; Ismail, H. Effect of acetylation and coupling agent treatments upon biological degradation of plant fibre reinforced polyester composites. Polym. Test. 2000, 20, 65-75. [CrossRef]

250. Mokoena, M.A.; Djoković, V.; Luyt, A.S. Composites of linear low density polyethylene and short sisal fibres: The effects of peroxide treatment. J. Mater. Sci. 2004, 39, 3403-3412. [CrossRef]

251. Joseph, K.; Thomas, S.; Pavithran, C. Effect of chemical treatment on the tensile properties of short sisal fibre-reinforced polyethylene composites. Polymers 1996, 37, 5139-5149. [CrossRef] 
252. Ahmad, E.; Luyt, A.S. Effects of organic peroxide and polymer chain structure on morphology and thermal properties of sisal fibre reinforced polyethylene composites. Compos. Part A Appl. Sci. Manuf. 2012, 43, 703-710. [CrossRef]

253. Imoisili, P.E.; Jen, T.-C. Mechanical and water absorption behaviour of potassium permanganate $\left(\mathrm{KMnO}_{4}\right)$ treated plantain (Musa Paradisiacal) fibre/epoxy bio-composites. J. Mater. Res. Technol. 2020, 9, 8705-8713. [CrossRef]

254. Zaman, H.U.; Khan, M.A.; Khan, R.A.; Rahman, M.A.; Das, L.R.; Mamun, A. Role of potassium permanganate and urea on the improvement of the mechanical properties of jute polypropylene composites. Fibers Polym. 2010, 11, 455-463. [CrossRef]

255. Li, W.; Meng, L.; Ma, R. Effect of surface treatment with potassium permanganate on ultra-high molecular weight polyethylene fiber reinforced natural rubber composites. Polym. Test. 2016, 55, 10-16. [CrossRef]

256. Mohammed, A.A.; Bachtiar, D.; Rejab, M.R.M.; Hasany, S.F. Effect of potassium permanganate on tensile properties of sugar palm fibre reinforced thermoplastic polyurethane. Indian J. Sci. Technol. 2017, 10, 1-5. [CrossRef]

257. Barkoula, N.M.; Alcock, B.; Cabrera, N.O.; Peijs, T. Flame-retardancy properties of intumescent ammonium poly(phosphate) and mineral filler magnesium hydroxide in combination with graphene. Polym. Polym. Compos. 2008, 16, 101-113. [CrossRef]

258. Orue, A.; Jauregi, A.; Unsuain, U.; Labidi, J.; Eceiza, A.; Arbelaiz, A.; Mendizabal, A.O. The effect of alkaline and silane treatments on mechanical properties and breakage of sisal fibers and poly(lactic acid)/sisal fiber composites. Compos. Part A Appl. Sci. Manuf. 2016, 84, 186-195. [CrossRef]

259. Pickering, K.; Abdalla, A.; Ji, C.; McDonald, A.; Franich, R. The effect of silane coupling agents on radiata pine fibre for use in thermoplastic matrix composites. Compos. Part A Appl. Sci. Manuf. 2003, 34, 915-926. [CrossRef]

260. Bilba, k.; Arsene, M.A. Silane treatment of Bagasse fiber for reinforcement of cementitious composites. Compos. Part A Appl. Sci. Manuf. 2008, 39, 1488-1495. [CrossRef]

261. Xie, Y.; Hill, C.A.; Xiao, Z.; Militz, H.; Mai, C. Silane coupling agents used for natural fiber/polymer composites: A review. Compos. Part A Appl. Sci. Manuf. 2010, 41, 806-819. [CrossRef]

262. Zhou, F.; Cheng, G.; Jiang, B. Effect of silane treatment on microstructure of sisal fibers. Appl. Surf. Sci. 2014, 292, 806-812. [CrossRef]

263. Hong, C.; Hwang, I.; Kim, N.; Park, D.; Hwang, B.; Nah, C. Mechanical properties of silanized jute-polypropylene composites. J. Ind. Eng. Chem. 2008, 14, 71-76. [CrossRef]

264. Asim, M.; Jawaid, M.; Abdan, K.; Ishak, M.R. The effect of silane treated fibre loading on mechanical properties of pineapple leaf/kenaf fibre filler phenolic composites. J. Polym. Environ. 2017, 26, 1520-1527. [CrossRef]

265. Kalia, S.; Kumar, A. Surface Modification of sunn hemp fibers using acrylation, peroxide and permanganate treatments: A study of morphology, thermal stability and crystallinity. Polym. Technol. Eng. 2013, 52, 24-29. [CrossRef]

266. Sampathkumar, D.; Punyamurthy, R.; Bennehalli, B.; Ranganagowda, R.P.; Chikkol Venkateshappa, S. Natural areca fibre: Surface modification and spectral studies type (method/approach) spectral studies council for innovative research. J. Adv. Chem. 2014, 10, 3263-3273.

267. Qin, L.; Qiu, J.; Liu, M.; Ding, S.; Shao, L.; Lü, S.; Zhang, G.; Zhao, Y.; Fu, X.; Lü, S. Mechanical and thermal properties of poly(lactic acid) composites with rice straw fiber modified by poly(butyl acrylate). Chem. Eng. J. 2011, 166, 772-778. [CrossRef]

268. Haque, R.; Saxena, M.; Shit, S.C.; Asokan, P. Fibre-matrix adhesion and properties evaluation of sisal polymer composite. Fibers Polym. 2015, 16, 146-152. [CrossRef]

269. Jawaid, M.; Othman, A.; Saba, N.; Shekeil, Y.A.; Paridah, M.T.; Abdul Khalil, H.P.S. Effect of chemical modifications of fibers on tensile properties of epoxy hybrid composites. Int. J. Polym. Anal. Charact. 2014, 19, 391-403. [CrossRef]

270. Mishra, S.; Misra, M.; Tripathy, S.S.; Nayak, S.K.; Mohanty, A.K. Graft copolymerization of acrylonitrile on chemically modified sisal fibres. Macromol. Mater. Eng. 2001, 286, 107-113. [CrossRef]

271. Thakur, V.K.; Kessler, M.R. Synthesis and characterization of AN-g-SOY for sustainable polymer composites. ACS Sustain. Chem. Eng. 2014, 2, 2454-2460. [CrossRef] 
272. Maniruzzaman, M.; Rahman, M.; Gafur, M.; Fabritius, H.; Raabe, D. Modification of pineapple leaf fibers and graft copolymerization of acrylonitrile onto modified fibers. J. Compos. Mater. 2011, 46, 79-90. [CrossRef]

273. Ray, D.; Bose, N.; Mohanty, A.K.; Misra, M. Modification of the dynamic damping behaviour of jute/vinylester composites with latex interlayer. Compos. Part B Eng. 2007, 38, 380-385. [CrossRef]

274. George, J.; Ivens, J.; Verpoest, I. Surface modification to improve the impact performance of natural fibre composites. In Proceedings of the International Conference on Composite Materials (ICCM), Paris, France, 5-9 July 1999.

275. Lee, J.; Bhattacharyya, D.; Zhang, M.; Yuan, Y. Mechanical properties of a self-healing fibre reinforced epoxy composites. Compos. Part B Eng. 2015, 78, 515-519. [CrossRef]

276. Gurunathan, T.; Mohanty, S.; Nayak, S.K. A review of the recent developments in biocomposites based on natural fibres and their application perspectives. Compos. Part A Appl. Sci. Manuf. 2015, 77, 1-25. [CrossRef]

277. Salazar, V.L.P.; Leão, A.L.; Rosa, D.D.S.; Gomez, J.G.C.; Alli, R.C.P. Biodegradation of coir and sisal applied in the automotive industry. J. Polym. Environ. 2011, 19, 677-688. [CrossRef]

278. Salimon, J.; Salih, N.; Yousif, E. Biolubricants: Raw materials, chemical modifications and Environmental benefits. Eur. J. Lipid Sci. Technol. 2010, 112, 519-530. [CrossRef]

279. Nassar, M.M.A.; Arunachalam, R.; Alzebdeh, K.I. Machinability of natural fibre reinforced composites: A review. Int. J. Adv. Manuf. Technol. 2017, 88, 2985-3004. [CrossRef]

280. Khaldi, N.; Vivet, A.; Bourmaud, A.; Sereir, Z.; Kada, B. Damage analysis of composites reinforced with Alfa fibres: Viscoelastic behavior and debonding at the fibre/matrix interface. J. Appl. Polym. Sci. 2016, 133, 43760. [CrossRef]

281. Ciccarelli, L.; Cloppenburg, F.; Ramaswamy, S.; Lomov, S.V.; Van Vuure, A.; Hong, N.V.; Thanh, T.C.; Tri, N.M.; Thomas, G. Sustainable composites: Processing of coir fibres and application in hybrid-fibre composites. J. Compos. Mater. 2019, 54, 1947-1960. [CrossRef]

282. Sassoni, E.; Manzi, S.; Motori, A.; Montecchi, M.; Canti, M. Novel sustainable hemp-based composites for application in the building industry: Physical, thermal and mechanical characterization. Energy Build. 2014, 77, 219-226. [CrossRef]

283. Rwawiire, S.; Tomkova, B.; Militky, J.; Jabbar, A.; Kale, B.M. Development of a biocomposite based on green epoxy polymer and natural cellulose fabric (bark cloth) for automotive instrument panel applications. Compos. Part B Eng. 2015, 81, 149-157. [CrossRef]

284. Kumar, R.; Haq, M.I.U.; Raina, A.; Anand, A. Industrial applications of natural fibre-reinforced polymer composites challenges and opportunities. Int. J. Sustain. Eng. 2018, 12, 212-220. [CrossRef]

285. Davoodi, M.; Sapuan, S.; Ahmad, D.; Aidy, A.; Khalina, A.; Jonoobi, M. Concept selection of car bumper beam with developed hybrid bio-composite material. Mater. Des. 2011, 32, 4857-4865. [CrossRef]

286. Graupner, N.; Herrmann, A.S.; Müssig, J. Natural and man-made cellulose fibre-reinforced poly(lactic acid) (PLA) composites: An overview about mechanical characteristics and application areas. Compos. Part A Appl. Sci. Manuf. 2009, 40, 810-821. [CrossRef]

287. Holbery, J.; Houston, D. Natural-fibre-reinforced polymer composites in automotive applications. JOM 2006, 58, 80-86. [CrossRef]

288. Suddell, B.C. Industrial fibres: Recent and current developments. In Proceedings of the Symposium on Natural Fibres, Rome, Italy, 20 October 2008; Volume 20, pp. 71-82.

289. Pickering, K. Properties and Performance of Natural-Fibre Composites; Elsevier: Amsterdam, The Netherlands, 2008.

290. Carus, M.; Eder, A.; Dammer, L.; Korte, H.; Scholz, L.; Essel, R.; Breitmayer, E.; Barth, M. Wood-Plastic Composites (WPC) and Natural Fibre Composites (NFC); Nova-Institute Hürth, Ger.: Hürth, Germany, 2015; p. 16.

291. GLOBAL HEMPß Automotive Composites. Available online: http://www.globalhemp.com/2011/02/automotivecomposites.html?fbclid=IwAR2b9-_29CctnHN2pm9iVWn6rp6X-ET0P_8PjHq1T2nfSTBwtft6LqC0AKQ (accessed on 14 September 2020).

292. Fan, M.; Fu, F. Advanced High Strength Natural Fibre Composites in Construction. In Woodhead Publishing Series in Composites Science and Engineering; Woodhead Publishing: Cambridge, UK, 2016.

293. Uddin, N. Developments in Fibre-Reinforced Polymer (FRP) Composites for Civil Engineering; Elsevier: Amsterdam, The Netherlands, 2013.

294. John, M.J.; Thomas, S. Biofibres and biocomposites. Carbohydr. Polym. 2008, 71, 343-364. [CrossRef]

295. Van De Weyenberg, I.; Ivens, J.; De Coster, A.; Kino, B.; Baetens, E.; Verpoest, I. Influence of processing and chemical treatment of flax fibres on their composites. Compos. Sci. Technol. 2003, 63, 1241-1246. [CrossRef] 
296. Ticoalu, A.; Aravinthan, T.; Cardona, F. A review of current development in natural fibre composites for structural and infrastructure applications. In Proceedings of the Southern Region Engineering Conference (SREC 2010), Toowoomba, Australia, 10-12 November 2010; pp. 113-117.

297. Sen, T.; Reddy, H.N.J. Various industrial applications of hemp, kinaf, flax and ramie natural fibres. Int. J. Innov. Manag. Technol. 2011, 2, 192-199.

298. Bongarde, U.S.; Shinde, V.D. Review on natural fibre reinforcement polymer composites. Int. J. Eng. Sci. Innov. Technol. 2014, 3, 431-436.

299. Mwaikambo, L. Review of the history, properties and application of plant fibres. Afr. J. Sci. Technol. 2006, 7, 120-133.

Publisher's Note: MDPI stays neutral with regard to jurisdictional claims in published maps and institutional affiliations.

(C) 2020 by the authors. Licensee MDPI, Basel, Switzerland. This article is an open access article distributed under the terms and conditions of the Creative Commons Attribution (CC BY) license (http://creativecommons.org/licenses/by/4.0/). 